\title{
Vortical Structures and Turbulent Bursts Behind Magnetic Obstacles in Transitional Flow Regimes
}

\author{
S. Kenjereš ${ }^{1}$, S. ten Cate and C. J. Voesenek \\ Department of Multi-Scale Physics, Faculty of Applied Sciences and J. M. Burgerscentre for Fluid \\ Dynamics, Delft University of Technology, \\ Prins Bernhardlaan 6, 2628 BW Delft, The Netherlands
}

\begin{abstract}
The present paper reports on numerical investigations of vortical structures in transient flow regimes generated by the local action of the Lorentz force on an electrically conductive fluid. The locally imposed non-uniform magnetic field generates similar effects as observed for flows over submerged solid obstacles. It is demonstrated that complex flow patterns can be generated by imposing magnetic fields of different strengths. The initial validation of the electromagnetically extended Navier-Stokes solver on unstructured numerical grids is performed in the low-Reynolds number range $100 \leq R e \leq 400$ for different values of the magnetic interaction parameter. A generally good agreement is obtained in comparison with similar numerical studies of Votyakov et al. $(2007,2008)$ for the low-Reynolds number cases. Then, a series of simulations are performed in transitional flow regimes $(R e=900)$ for different values of the interaction parameter $(N=3, \ldots, 25)$. Simulations demonstrated the appearance of vortex-shedding phenomena similar to the flows behind solid obstacles. In contrast to the solid obstacles, the magnetic obstacles also generated the vortical flow patterns inside the magnetically affected regions. This feature can be used for the flow control of electrically conductive fluids, for efficient enhancements of the wallheat transfer or for better mixing of passive scalars. Despite the laminar inflow conditions, turbulent bursts are observed in the magnetic wake region for the $R e=900$ case. The velocity spectra and spatial distributions of the long-time averaged second-moments of the velocity field demonstrated that turbulence was locally sustained in the proximity of the magnetic wake edge.
\end{abstract}

Keywords: magnetic obstacle, Lorentz force, vortex shedding, turbulent bursts

\footnotetext{
${ }^{1}$ Corresponding author. Tel.:+31-15-2783649; Fax.:+31-15-2782838; E-mail address: S.Kenjeres@tudelft.nl
} 


\section{Introduction}

The predictions of electrically conductive fluid motion in transient and turbulent regimes in the presence of an imposed non-homogeneous magnetic field is the key for the potential optimisation studies of numerous industrial and technological processes. Such interactions between flow and electromagnetic field (EMF) are present in metallurgy (continuous steel casting, arc welding for joining metals, electromagnetic stirring and mixing), semiconductor production (crystal growth in steady, fixed and rotating magnetic fields), aerospace (hypersonic aerodynamic control and thrust vectoring by magnetohydrodynamics (MHD) devices), or in the new generation of fusion reactors (International Thermonuclear Experimental Reactor (ITER) project). Since the experiments in such extreme conditions are difficult to perform, numerical insights into flow/turbulence/EMF interactions can provide important information that can be used for control and further optimisations in a wide range of applications.

The present study explores similarity concepts between flows around solid and magnetic obstacles. Due to the active Lorentz force, a multi-vortex pattern can occur in the wake of a magnetic obstacle, Fig. 1. In addition to the wake region, strong flow reorganisation is also present inside the magnetic obstacle. This feature makes it possible to enhance mixing or heat transfer. The two-dimensional studies of the flow past a magnetic obstacle for very low $R e(R e=100$ and 200) were studied in Cuevas et al. (2006a, 2006b) and Beltran et al. (2010). It was shown that after reaching a critical threshold of imposed magnetic field, the instantaneous velocity field revealed the appearance of vortex shedding behind a magnetic obstacle. Votyakov et al. $(2007,2008)$ reported on a three-dimensional constrained flow around a magnetic obstacle in a rectangular duct. The $100 \leq R e \leq 400$ range was considered in this study and different aspect ratios of the imposed magnetic obstacle analysed. Stationary flow fields were obtained and analysed in the central horizontal plane. Votyakov and Kassinos (2009) reported on the analogy between streamlined magnetic and solid obstacles. They observed that for a Reynolds number larger than a critical value, the braking of vortices (vortex shedding) from the magnetic obstacle was similar to those occurring past solid obstacles. Additional analysis of the core of the magnetic obstacle was performed in Votyakov and Kassinos (2010). The two-dimensional numerical simulations revealed a new recirculation patterns composed of many (even number) vortices aligned along the spanwise line crossing the magnetic gap.

The present study focuses on the generation of vortical structures by imposed magnetic obstacles in the transitional flow regimes. Spatial and temporal evolutions of turbulent bursts, identified as pockets of high velocity fluctuations, will be analysed in details. 


\section{Mathematical formulation}

The equations describing interactions between an imposed steady magnetic field and a flow of an incompressible, electrically conductive fluid consist of conservation of mass, momentum and total electric current, and is finally closed by an additional equation for electric potential, originating from Ohm's law for a moving conductor:

$$
\begin{gathered}
\nabla \cdot \mathbf{V}=0, \quad \nabla \cdot \mathbf{B}=0, \quad \nabla \cdot \mathbf{J}=0 \\
\rho\left[\frac{\partial \mathbf{V}}{\partial t}+(\mathbf{V} \cdot \nabla) \mathbf{V}\right]=-\nabla p+\mu \nabla^{2} \mathbf{V}+\mathbf{J} \times \mathbf{B} \\
\mathbf{J}=\sigma(-\nabla \phi+\mathbf{V} \times \mathbf{B}), \quad \nabla^{2} \phi=\nabla \cdot(\mathbf{V} \times \mathbf{B})
\end{gathered}
$$

Here, $\mathbf{V}, \mathbf{B}, \mathbf{J}$ and $\phi$ are the velocity, the magnetic field, the total electric current and the electric potential, respectively, Kenjereš and Hanjalić (2000). The strength of interactions between the fluid and the electromagnetic field is described by the interaction parameter $N$ that represents a ratio between the Lorentz and the inertial forces, i.e. $N=\sigma L_{0} B_{0}^{2} / \rho V_{0}$, where $\sigma$ is the electric conductivity, $\rho$ is the density of the fluid and $B_{0}$ is the magnetic field intensity in the centre of the magnetic gap. The Reynolds number is defined as $R e=V_{0} L_{0} / \nu$, where $V_{0}$ is the bulk horizontal inlet velocity, $L_{0}$ the vertical half-distance of the rectangular duct (in the y-direction) and $\nu$ the kinematic viscosity. The Hartmann number represents a ratio between the Lorentz and the viscous forces, $H a=B_{0} L_{0} \sqrt{\sigma / \rho \nu}=\sqrt{N R e}$. The magnetic field distributions originating from permanent magnets of opposite polarities and dimensions $-M_{x} \leq x \leq M_{x}$, $-M_{z} \leq z \leq M_{z}$, with separation between magnets $-h \leq y \leq h$ and enclosed in a ferromagnetic yoke, can be calculated from a semi-analytical simplification of the Biot-Savart's and Maxwell equations, Akoun et al. (1984), Votyakov et al. (2008, 2009):

$$
\begin{array}{r}
B_{x}(x, y, z)=\sum_{k= \pm 1} \sum_{j= \pm 1} \sum_{i= \pm 1}(i j k) \operatorname{arctanh}\left(\frac{z-k M_{z}}{r(i, j, k)}\right) \\
B_{z}(x, y, z)=\sum_{k= \pm 1} \sum_{j= \pm 1} \sum_{i= \pm 1}(i j k) \operatorname{arctanh}\left(\frac{x-i M_{x}}{r(i, j, k)}\right) \\
B_{y}(x, y, z)=-\sum_{k= \pm 1} \sum_{j= \pm 1} \sum_{i= \pm 1}(i j k) \arctan \left(\frac{\left(x-i M_{x}\right)\left(z-k M_{z}\right)}{(y-j h) r(i, j, k)}\right)
\end{array}
$$

where $r=\sqrt{\left(x-i M_{x}\right)^{2}+(y-j h)^{2}+\left(z-k M_{z}\right)^{2}}$. The field is then multiplied such that $\mathbf{B}(0,0,0)=$ $B_{0} \mathbf{y}$, where $B_{0}$ was selected to obtain desired $N$ value. With known components of the imposed magnetic field, we have a fully closed system of equations, Eqs.(1-3). Note that this system of equations is valid only for the one-way coupling between velocity and electromagnetic fields (no backreaction of the velocity field on the imposed magnetic field, i.e. when the magnetic Reynolds number 
$R e_{m}=V_{0} L_{o} / \lambda<<1$ (Kenjereš et al., 2006; Kenjereš and Hanjalić, 2007a, 2007b; Kenjereš, 2009b). Despite the one-way coupling between velocity and imposed magnetic field (the imposed magnetic field is constant in time), the Lorentz force is both space- and time-dependent (in contrast to situations where the imposed Lorentz force was constant in time, e.g. Thibault and Rossi, 2003; Rossi et al., 2006a, 2006b; Kenjereš et al., 2009; Kenjereš, 2008, 2009a, 2011). This is because of the relatively high electric conductivity of the fluid where also induced electric current must be taken into account (similar to Hanjalić and Kenjereš, 2000, 2001; Kenjereš and Hanjalić, 2004 - but where a uniformly imposed magnetic field was applied over the entire flow domain). The direct influence of the Lorentz force $(\mathbf{F}=\mathbf{J} \times \mathbf{B})$ will be confined to the region where the magnetic field is present (inside the magnetic obstacle).

\section{Numerical method}

The system of equations (1)-(3) is discretised and solved by a finite-volume based numerical solver for unstructured numerical grids, Ničeno et al. (2005). The numerical code is able to combine hexagonal and tetrahedral control volumes (CVs), but since the presently simulated flows are in a simple rectangular geometry, a structured non-uniform rectangular mesh is employed. The mesh is refined in spanwise direction in the proximity of the walls and in streamwise direction in the proximity of the magnetic obstacle edges (the entrance and the outlet of the magnetic field region). Convective and diffusive terms are calculated by the second-order central-difference scheme (CDS). Time integration is performed using a fully implicit second-order three-consecutive-time levels scheme. The value of the time step is set to provide that $\mathrm{CFL} \approx 0.5$. The SIMPLE algorithm is used for coupling velocity and pressure fields. The linearised system of discretised equations is solved by the diagonally incomplete LU pre-conditioned Cholesky Bi-Conjugate Gradient method (BiCG), Barrett et al. (1994). The message-passing-interface (MPI) domain-decomposition directives are used to run solver in the parallel mode. The present simulations were performed using 4-8 CPUs on the local Beowulf-Linux cluster. 


\section{Results}

\subsection{Low Reynolds number range $(100 \leq R e \leq 400)$}

The flow of an electrically conductive fluid (Galinstan, $\mathrm{Ga}_{0.68} \operatorname{In}_{0.20} \mathrm{Sn}_{0.12}, \rho=6360 \mathrm{~kg} / \mathrm{m}^{3}, \mu=2.1624 \times 10^{-3}$ $\mathrm{Pa} \cdot \mathrm{s}, \sigma=3.46 \times 10^{6} \mathrm{~S} / \mathrm{m}$ ) in a rectangular channel with electrically insulated walls subjected to a nonuniform magnetic field in the low-Reynolds number flow regimes $(100 \leq R e \leq 400)$ is considered first, Fig. 2. The geometrical parameters are adjusted to be similar with the parameters used in Votyakov et al. $(2007,2008)$ in order to validate extensions of the Navier-Stokes solver with additional equations for electrical potential and magnetic field, and, consequently, with additional Lorentz force in the momentum equation, Eqs.(1)-(3). A numerical mesh of $128^{3} \mathrm{CVs}$ is employed with local refinements in the proximity of the walls and the magnetic edges (the entrance and the outlet of the magnetic field region).

The inflow conditions are specified from a fully-developed laminar flow in a rectangular duct. Noslip boundary conditions are applied for all velocity components at all walls. The electrically insulated walls are imposed by specifying a zero-gradient of the electric potential $\left(\partial \phi / \partial x_{n}=0\right)$ to ensure zero current through the wall $\left(\mathbf{J}_{\mathbf{w}}=0\right)$. The far-field (zero gradient) condition is imposed at the outlet for all variables.

The external magnetic field is imposed by solving equations (4) with following parameters: $\left(M_{x}, h\right.$, $\left.M_{z}\right)=(0.015,0.015,0.02) \mathrm{m}$. The strength of the imposed magnetic field $B_{0}$ was adjusted in order to get proper values of the interaction parameter $N$. The magnetic constrainment factor that defines the ratio between the spanwise extension of the magnetic field and the spanwise channel length is $\kappa=M_{z} / L_{z}=0.4$. Distributions of the magnetic field lines in characteristic planes are shown in Fig. 3.

The calculated flow patterns are shown in Fig. 4. Steady solutions are obtained for all considered cases. The appearance of vortical flow patterns occurs after reaching a critical value of the interaction parameter $(N)$, depending on the value of the Reynolds number. For $R e=100$ and the low interaction parameter case $N=4, H a=20$, the magnetic field deflects the approaching fluid towards the side walls causing its acceleration and creation of two side-wall jets, Fig. 4-top. Due to the relatively weak Lorentz force suppression in the magnetic obstacle region, no recirculative regions are created. With further increase of $N$ to 11.25 ( $H a=33.5)$, a vortex-pair is generated inside the magnetic obstacle area, Fig. 4middle. By keeping $N=11.25$ and increasing the Reynolds number to $R e=400(H a=67)$, additional counter-rotating vortex pairs are generated in the magnetic wake region, Fig. 4-bottom. Identical reor- 
ganisation of flow structures was presented in Votyakov et al. (2007, 2008). Since different numerical discretisation methods (the finite-volume versus the finite-differences approach of Votyakov et al.) and different numerical mesh are used, we performed detailed comparisons with the results of Votyakov et al. $(2007,2008)$.

The non-dimensionalised streamwise velocity profiles (normalised to maximum inlet velocity) along the centreline are shown in Fig. 5. The characteristic velocity used for non-dimensionalisation was the incoming area-averaged streamwise velocity $V_{0}$. It can be seen that a very good agreement is obtained for cases $\mathrm{A}$ and $\mathrm{B}$ - both in the front and behind the magnetic obstacle. Comparison for the case $\mathrm{C}$ shows some deviations - especially in the magnetic wake region. While both simulations predicted three vortex pairs, the length of the recirculation defined by the horizontal extension of the third vortex pair is different. This can be a consequence of the different mesh densities used in the wake region - where the presented simulations employed a significantly finer mesh in this region than that used in Votyakov et al. $(2007,2008)$ (where the finest mesh was $\left.64^{3}\right)$. Note also that we simulated a longer domain than used in Votyakov et al. in order to allow the post-magnetic recovery of the flow. This longer domain is also needed to properly capture the transient effects for the higher values of $R e$. It can be concluded that generally good agreement between the present results and results of Votyakov et al. (2007) is obtained for the considered range of the low Reynolds numbers and interaction parameters, and that steady laminar flow patterns are obtained.

\subsection{Intermediate Reynolds number range $(R e=900)$}

Votyakov and Kassinos (2009) observed vortex shedding past a magnetic obstacle at $R e=900, N=9$. They studied two kinds of initial and inlet conditions: unperturbed and perturbed case. For the unperturbed symmetric inlet velocity a symmetric vortex shedding was observed. For the perturbed inlet velocity, an asymmetric vortex shedding occurs.

To be able to access higher values of $R e$ and $N$ than previously addressed, the configuration shown in Fig. 2 is adjusted. In order to reduce numerical resolution requirements imposed by the presence of the four walls (since both hydrodynamical and Hartmann boundary layers must be properly resolved in the proximity of the walls), we will consider a configuration where the two-side walls are removed and replaced by symmetry boundary conditions (i.e. walls in the z-directions are removed). A new numerical mesh was created consisting of $240 \times 80 \times 100$ control volumes that are refined in the proximity of the 
remaining walls and edges of the imposed magnetic field. This numerical mesh should be sufficiently fine to properly resolve the flow for the selected value of $R e=900 .^{2}$

\subsubsection{Instantaneous features}

In order to portray the electromagnetic field distributions for the transient cases, lines of the total electric current $(\mathbf{J})$ and contours of the electric potential $(\phi)$ in the central horizontal plane are shown in Fig. 6. Note that distributions of the electric potential, and consequently, of the total current are different for various values of $N$. This is because of the mutual interdependency between velocity and electromagnetic fields. Since the cross product of the velocity and imposed magnetic field $(\mathbf{V} \times \mathbf{B})$ is the source term in the Poisson scalar equation of the electrical potential Eq.(3), any change in the velocity field (V) will influence the distribution of the electric potential $(\phi)$. This change in the electric potential will lead to changes of the total electric current $(\mathbf{J})$ and finally, through the Lorentz force effects $\left(\mathbf{F}^{\mathbf{L}}=\mathbf{J} \times \mathbf{B}\right)$, the underlying velocity field will be affected, and a full circle of interactions will be closed. It can be seen that a stronger magnetic field generates stronger electric potential differences, and consequently, stronger electric currents, Fig. 6. The stronger total electric current generates a stronger Lorentz force and imposes a stronger braking of the flow.

Time evolutions of the flow instabilities for a fixed value of $R e=900$ and two values of the interaction parameter $(N=3$ and $10 ; H a=52$ and 95) are shown in Figs. 7 and 8. The characteristic flow-through time for these particular parameters is $t^{*}=20 \mathrm{sec}$. The instantaneous contours of the non-dimensional streamwise velocity are shown in the central horizontal plane $(y=0 \mathrm{~m})$. The action of the Lorentz force is already visible in the initial stage of the flow development. The strong flow suppression occurs in the centre of the magnetic obstacle. Then, as time progresses, the wake region is created and strong shear $\partial V_{x} / \partial z$ is generated between the slowly moving core and accelerated side jets. This leads to an elongated wake structure, which after reaching a critical length, starts to oscillate in the form of periodic vortex shedding. The dynamics of the development of the flow instabilities is similar for both values of the interaction parameters. The most striking differences are in the existence of the flow reversal region in the magnetic wake for $N=10$ case, as well as in the streamwise extension of the magnetic wake. The

\footnotetext{
${ }^{2}$ The Kolmogorov length scales were estimated from $\eta=\left(\nu^{3} / \varepsilon\right)^{1 / 4}$, where the dissipation rate of the turbulent kinetic energy is evaluated as $\varepsilon \approx V_{0}^{3} / L$, giving $\eta \approx 6 \cdot 10^{-5} \mathrm{~m}$. The used numerical mesh satisfies this requirement inside the magnetic obstacle and in the proximity of the magnetic edges. Further away, it is in $2 \eta \leq \Delta V^{1 / 3} \leq 5 \eta$ range, where $\Delta V=$ $\Delta x \cdot \Delta y \cdot \Delta z$ is volume of the numerical cell.
} 
higher value of the interaction parameter $(N=10)$ generates a significantly shorter magnetic wake, Fig. 8 .

For this particular value of Reynolds number $(R e=900)$, the inner and connecting vortex pairs are generated for $N \geq 5$, Fig. 9. These vortex pairs are very stable in time and only the attached vortex pair is highly intermittent. It can be seen that the vortex shedding effects are triggered by instabilities of the attached vortex pair. For all our simulations, symmetrical fully developed profiles were imposed - without any additional perturbations which were imposed in Votyakov and Kassinos (2009) - and asymmetric patterns emerged. In order to confirm the flow stability inside and in the proximity of the magnetic obstacle region, profiles of the instantaneous streamwise velocity along the $(x, y)=(0.07,0$. $) \mathrm{m}$ line for different interaction parameters are depicted in Fig. 9-top.

The side jets are caused by a local flow deceleration in the central part due to the magnetic obstacle - since the mass conservation must be satisfied. The spanwise velocity components exhibit a fully symmetrical behaviour with respect to the centreline, Fig. 9-bottom. The higher peaks are again associated with stronger magnetic damping of the flow in the central region. The profiles of the velocity components at an arbitrary time instant along the centreline for different values of $N$ are plotted in Fig. 10. Three characteristic regions can be distinguished: the region from the inlet till the magnetic centre - characterised by strong suppression of the flow and appearance of flow reversal (for higher $N$ ), a magnetic wake region - a stable region characterised by low velocity, and finally, a highly intermittent region characterised by strong fluctuations of all velocity components. Now we will analyse time series of velocity components at two monitoring locations behind the magnetic obstacle for different values of $3 \leq N \leq 25$ : inside the magnetic wake region, MON1 $(0.15,0 ., 0$. $) \mathrm{m}$, Fig. 11 , and at the edge of the magnetic wake region MON2 $(0.35,0 ., 0.) \mathrm{m}$, Fig. 12 . As previously discussed, the velocity fluctuations are significantly stronger for the second location. Whereas the first monitoring location is just behind the second vortex pair that is quite stable in time, the second monitoring location lies in the region of the influence of the initially stable third vortex pair. As time progresses, this third vortex pair starts to be elongated in the spanwise direction and the flow separates. This is a very intermittent mechanism that triggers the generation of additional eddies that are carried by the underlying flow in the streamwise direction. Note the appearance of the transient bursts at $t=125 \mathrm{sec}$, Fig. 12. This is the moment when the break-up of the attached vortex pair occurs.

The power spectra of the non-dimensional velocity components at two monitoring locations and for two selected values of the interaction parameter, $N=3$ and 15, are shown in Figs. 13 and 14. The appearance of the distinct peak frequency of the spanwise velocity component is already visible at the 
first monitoring location, Fig. 13. This characteristic peak is strongly increased at the second monitoring location, Fig. 14. It is interesting to observe that the actual value of the vortex shedding frequency of $f=0.11 \mathrm{~Hz}$ is identical for both values of the interaction parameter. This gives following value of the Strouhal number, $S t=f D / V_{0}=0.144$, where $D$ is the cross-stream size of the magnet $\left(D=2 M_{z}\right)$. For their two-dimensional simulations, Cuevas et al. (2006b) observed the characteristic value of $S t=0.1$. Similarly, for three-dimensional simulations, Votyakov and Kassinos (2009) reported that $S t=0.25$.

For both values of the interaction parameter, the energy of the fluctuations is the smallest for the vertical velocity component at the first monitoring location, Fig. 13. This is changed for the second monitoring location where the vertical velocity components is now overtaking its spanwise component for $N=15$ case, Fig. 14-bottom. It indicates the development of the three-dimensional instabilities. The Kolmogorov inertial range with characteristic $-5 / 3$ slope is observed for the second monitoring location and $N=15$. It proves the localised state of the developed turbulence at this location, Fig. 14-bottom. To provide more insights into turbulence behaviour and its spatial redistribution, the second-order moments of the velocity field are analysed next.

\subsubsection{Long-time averaged fields}

Now we focus on the long-time averaged velocity fields. After reaching a fully developed flow with distinct vortex-shedding structures, we started to collect statistics for evaluation of the first- and secondorder moments. In order to get fully statistically convergent averaging samples, we used $10^{4}$ instantaneous realisations. Three-dimensional representations of the long-term averaged velocity field for $R e=900$ and for two different values of the interaction parameter $N=3$ and 10, are shown in Fig. 15 . For the weak magnetic interaction parameter $(N=3)$, the averaged flow is slightly deflected from the magnetic region and accelerated in the spanwise direction $(z-)$, but there is no visible recirculation, Fig. 15-top. In contrast to that, for the intermediate interaction number $N=10$, both the inner and connecting vortex pairs are generated in the averaged flow field, Fig. 15-bottom. The contours of the mean streamwise and spanwise velocity components in the central horizontal plane $(y=0 \mathrm{~m})$ are shown in Fig. 16. The length of the magnetic wake extension is quite different for considered values of the interaction parameters $(N=3$ and 10). A stronger imposed magnetic field generates a significantly shorter wake. The flow is also rapidly deflected and accelerated in the spanwise direction due to the magnetic blockage effect in the central part of the domain. This is also indicated by the contours of the normalised spanwise velocity component $\left(V_{z} / V_{0}\right)$ in Fig. 16. 
The turbulent activities are confined in distinct pockets of the flow where a strong shear takes place, Fig. 17. Note that the approaching flow is in the laminar flow regime $(R e=900)$. The turbulent kinetic energy has maximum values in the proximity of the edge of the wake extension where two side jets meet ( $x=0.55$ and $0.35 \mathrm{~m}$, for $N=3$ and 10 , respectively). Two expanding wakes (characterised by a low turbulence intensity) originating from the edges of the magnetic obstacle are observed for the low interaction parameter $(N=3)$, Fig. 17-top. These expanding wakes are not present for the higher value of the interaction parameter $(N=10)$, Fig. 17-bottom. After reaching a maximum, the turbulent kinetic energy is progressively decaying, indicating the flow relaminarisation farther downstream. Despite the appearance of the strong vortex shedding intermittency imposed by the magnetic obstacle, the turbulence is not able to be fully sustained farther downstream for the considered value of $R e=900$. A similar mechanism of vortex bursting in a laminar environment as a source of turbulence was addressed in experimental studies of Cuypers et al. (2003). In their studies, the initial vortex was generated by a small step obstacle that was added to a laminar boundary layer, and was strongly enhanced by sucking the flow though the slots on each lateral wall. By analysing the turbulent kinetic energy spectra at different locations inside the flow, they identified the existence of an inertial range turbulent behaviour, characterised by the $-5 / 3$ Kolmogorov law, at the location where the vortex bursts. To provide more insights into energy redistribution among the fluctuating velocity components inside the turbulent pockets, the turbulent stresses are shown in Figs. 18 and 19. For $N=3$ case, the streamwise turbulent stress is the largest, Fig. 18. Despite the zero mean value of the vertical velocity component $\left(V_{y} / V_{0}\right)$ at this location, the vertical turbulent stress $\left(\overline{v_{y} v_{y}} / V_{0}^{2}\right)$ is significant. It is smallest among the normal stress components due to the vertical confinement of the flow by channel walls. The normal turbulent stress components exhibit entirely different behaviour for the higher interaction parameter $(N=10)$, Fig. 19. Now, the spanwise turbulent stress is the largest $\left(\overline{v_{z} v_{z}}\right)$ whereas the vertical turbulent stress is the smallest $\left(\overline{v_{y} v_{y}}\right)$ indicating the redirection in the turbulent kinetic energy exchange. The fluctuations in the vertical direction $\left(\overline{v_{y} v_{y}}\right)$, although the smallest in intensity, occupy a larger part of domain compared to its streamwise and spanwise components, which are much more focused around the location where magnetic wake ends. The turbulent shear stress component also exhibit different behaviour for different values of $N$. For the low interaction number case $(N=3)$, two regions with high values of turbulent shear stress $\left(\overline{v_{x} v_{z}}\right)$ of opposite signs are symmetrically placed with respect to the centreline, Fig. 18. Additional regions with secondary peaks of high turbulent shear stress in both $x$ - and $z$-direction are present for the higher interaction $(N=10)$ case. 


\section{Summary and conclusions}

We performed numerical simulations of the flow of an electrically conductive fluid under the one-way coupled influence of a locally imposed non-uniform magnetic field. A fully coupled system of equations involves solving the Navier-Stokes equations for velocity and pressure fields, and a simplified set of the Maxwell's equations for distributions of the imposed magnetic field and generated electric currents. The velocity and electromagnetic fields are mutually coupled through the scalar equation of the electric potential, the total electric current and finally, through the action of the Lorentz force in the momentum equation. Detailed validation of the newly developed solver for fully-unstructured meshes is performed for the low Reynolds number flows $(100 \leq R e \leq 400)$ and intermediate values of the interaction parameter $4 \leq N \leq 11.25$. Generation of multi-vortex patterns inside and behind the magnetic field region is clearly demonstrated. Obtained results demonstrated a good agreement with available data presented in literature under similar conditions, Votyakov et al. (2007, 2008). Then, a series of simulations for an intermediate Reynolds number $(R e=900)$ and various values of the interaction parameter $(3 \leq N \leq 25)$ are performed. In contrast to the previous simulations for the lower Reynolds numbers that produced stable flow patterns, the $R e=900$ case produced vortex shedding. We have visualised the temporal dynamics of the vortical structures in the magnetic obstacle wake. Despite big differences of vortical flow structures inside the magnetic obstacle for different values of the interaction parameter $(N)$, the shedding frequency of the detached vortices was not affected by $N$. The velocity spectra at characteristic locations in the magnetic wake revealed imprints of fully-developed turbulence. By analysing the long-term averaged second-moments of the velocity fields, it is concluded that turbulence was locally sustained in the proximity of the magnetic wake edge. A rapid turbulence decay indicated that flow will fully relaminarise farther downstream.

\section{References}

Akoun, G. and Yonnet, J., P., 1984. 3D analytical calculation of the forces exerted between two cuboidal magnets. IEEE Trans. Magnet. 20, pp.1962-1964.

Barrett, R., Berry, M., Chan, T. F., Demmel, J., Donaro, J., Dongarra, J., Eijkhout, V., Pozo, R., Romine, C., Van der Vorst, H., 1994. Templates for the Solution of Linear Systems: Building Blocks for Iterative Methods, 2nd Edition, SIAM. 
Beltran, A., Ramos, E., Cuevas, S. and Brons, M., 2010. Bifurcation analysis in a vortex flow generated by an oscillatory magnetic obstacle. Phys. Rev. E. 81, 036309, pp.1-7.

Cuevas, S., Smolentsov, S. and Abdou, M. A., 2006a. On the flow past a magnetic obstacle. J. Fluid Mech. 553, pp.227-252.

Cuevas, S., Smolentsov, S. and Abdou, M. A., 2006b. Vorticity generation in creeping flow past a magnetic obstacle. Phys. Rev. E 74 (5), 056301, pp.1-10.

Cuypers, Y., Maurel, A., and Petitheans, P., 2003. Vortex Burst as a Source of Turbulence. Phys. Rev. Lett. 91, No. 19, Article 194502, pp. 1-4.

Hanjalić, K. and Kenjereš, S., 2000. Reorganization of turbulence structure in magnetic RayleighBénard convection: a T-RANS study. Journal of Turbulence 1 (8), pp. 1-22.

Hanjalić, K. and Kenjereš, S., 2001. T-RANS simulation of deterministic eddy structure in flows driven by thermal buoyancy and Lorentz force. Flow, Turbulence and Combustion 66 (4), pp. 427-451.

Kenjereš, S. and Hanjalić, K., 2000. On the implementation of effects of Lorentz force in turbulence closure models. Int. J. Heat and Fluid Flow 21 (3), pp. 329-337.

Kenjereš, S. and Hanjalić, K., 2004. Numerical simulation of magnetic control of heat transfer in thermal convection. Int. J. Heat and Fluid Flow 25 (3), pp. 559-568.

Kenjereš, S., Hanjalić, K., Renaudier, S., Stefani, F., Gerbeth, G. and Gailitis, A., 2006. Coupled fluid-flow and magnetic-field simulation of the Riga dynamo experiment. Physics of Plasmas 13 (12), Art. No. 122308, pp.1-14.

Kenjereš, S. and Hanjalić, K., 2007a. Numerical simulation of a turbulent magnetic dynamo. Physical Review Letters 98 (10), Art. No. 104501, pp.1-4.

Kenjereš, S. and Hanjalić, K., 2007b. Numerical insights into magnetic dynamo action in a turbulent regime. New Journal of Physics 9, Art. No. 306, pp. 1-29.

Kenjereš, S., 2008. Electromagnetic enhancement of turbulent heat transfer. Physical Review E 78, Art. No. 066309, pp. 1-5.

Kenjereš, S., 2009a. Large eddy simulations of targeted electromagnetic control of buoyancy-driven turbulent flow in a slender enclosure. Theoretical and Computational Fluid Dynamics 23 (6), pp.471- 
Kenjereš, S., 2009b. Hybrid simulations of two-way coupled turbulent magnetohydrodynamic flows. Int. J. Multiscale Computational Engineeeing 7 (6), pp.545-558.

Kenjereš, S., Verdoold, J., Tummers, M. J., Hanjalić, K. and Kleijn, C. R., 2009. Numerical and experimental study of electromagnetically driven vortical flows. Int. J. Heat and Fluid Flow 30 (3), pp.494-504.

Kenjereš, S., 2011. Electromagnetically driven dwarf tornados in turbulent convection. Physics of Fluids 23, 015103, pp.1-10.

Ničeno, B., Hanjalić, K. and Basara, B., 2005. Unstructured Large-Eddy Simulation for vehiclecomponents aerodynamics: flow over an idealised car mirror. Progress in Computational Fluid Dynamics 5 (8), pp.427-443.

Pier, B., 2002. On the frequency selection of finite-amplitude vortex shedding in the cylinder wake. J. Fluid Mech. 458, pp.407-417.

Rossi, L., Vassilicos, J. C. and Hardalupas, Y., 2006a. Electromagnetically controlled multi-scale flows. J. Fluid Mech. 558, pp.207-242.

Rossi, L., Vassilicos, J. C. and Hardalupas, Y., 2006b. Multiscale laminar flows with turbulentlike properties. Phys. Rev. Lett. 97 (14), 144501, pp.1-4.

Thibault, J. P. and Rossi, L., 2003. Electromagnetic flow control: characteristic numbers and flow regimes of a wall-normal actuator. Journal of Physics D - Applied Physics 36 (20), pp.2559-2568.

Votyakov, E. V., Kolesnikov, Yu., Andreev, O., Zienicke, E. and Thess, A., 2007. Structure of the Wake of a Magnetic Obstacle. Phys. Rev. Lett. 98, 144504, pp.1-4.

Votyakov, E. V., Zienicke, E. and Kolesnikov, Y. B., 2008. Constrained flow around a magnetic obstacle, J. Fluid Mech. 610, pp.131-156.

Votyakov, E. V., Kassions, S. C., Albert-Chico, X., 2009. Analytic models of heterogeneous magnetic fields for liquid metal flow simulations. Theoretical and Computational Fluid Dynamics 23 (6), pp.571578.

Votyakov, E. V. and Kassinos, S. C., 2009. On the analogy between streamlined magnetic and solid 
obstacles. Physics of Fluids 21, 097102, pp.1-11.

Votyakov, E. V. and Kassinos, S. C., 2010. Core of the magnetic obstacle. Journal of Turbulence 11 (49), pp.1-15. 


\section{Nomenclature}

\begin{tabular}{|c|c|}
\hline$B_{0}$ & magnetic field intensity in the centre $(\mathrm{T})$ \\
\hline$D, L_{0}$ & characteristic dimensions (m) \\
\hline$M_{x}, h, M_{z}$ & characteristic magnet dimensions (m) \\
\hline$V_{0}$ & characteristic velocity $(\mathrm{m} / \mathrm{s})$ \\
\hline$R e=\frac{V_{0} L_{0}}{\nu}$ & Reynolds number (-) \\
\hline$R e_{m}=\frac{V_{0} L_{0}}{\lambda}$ & magnetic Reynolds number (-) \\
\hline$N=\frac{\sigma B_{0}^{2} L_{0}}{\rho V_{0}}$ & magnetic interaction parameter (-) \\
\hline$H a=B_{0} L_{0} \sqrt{\frac{\sigma}{\rho \nu}}$ & Hartmann number (-) \\
\hline$S t=\frac{f D}{V_{0}}$ & Strouhal number (-) \\
\hline$p$ & Pressure $(\mathrm{Pa})$ \\
\hline$f$ & vortex shedding frequency $(\mathrm{Hz})$ \\
\hline V & velocity $(\mathrm{m} / \mathrm{s})$ \\
\hline B & magnetic field $(\mathrm{T})$ \\
\hline $\mathbf{E}$ & electric field intensity $(\mathrm{V} / \mathrm{m})$ \\
\hline $\mathbf{F}=\mathbf{J} \times \mathbf{B}$ & Lorentz force density $\left(\mathrm{N} / \mathrm{m}^{3}\right)$ \\
\hline $\mathbf{J}$ & total current density $\left(\mathrm{A} / \mathrm{m}^{2}\right)$ \\
\hline
\end{tabular}

\section{Greek symbols}

$\phi \quad$ electric potential (V)

$\rho$ fluid density $\left(\mathrm{kg} / \mathrm{m}^{3}\right)$

$\nu \quad$ kinematic viscosity $\left(\mathrm{m}^{2} / \mathrm{s}\right)$

$\lambda$ magnetic diffusivity $\left(\mathrm{m}^{2} / \mathrm{s}\right)$

$\sigma$ electric conductivity (S/m)

$\kappa$ magnetic constrainment factor (-) 
Table 1: Overview of simulated configurations.

\begin{tabular}{|c|c|c|c|}
\hline \hline$R e[-]$ & $N[-]$ & $H a[-]$ & $\left|B_{0}\right|[T]$ \\
\hline 100 & 4. & 20. & 0.05 \\
100 & 11.25 & 33.5 & 0.084 \\
400 & 11.25 & 67. & 0.168 \\
900 & 3. & 52. & 0.13 \\
900 & 10. & 95. & 0.24 \\
900 & 15. & 116. & 0.3 \\
900 & 25. & 150. & 0.375 \\
\hline \hline
\end{tabular}



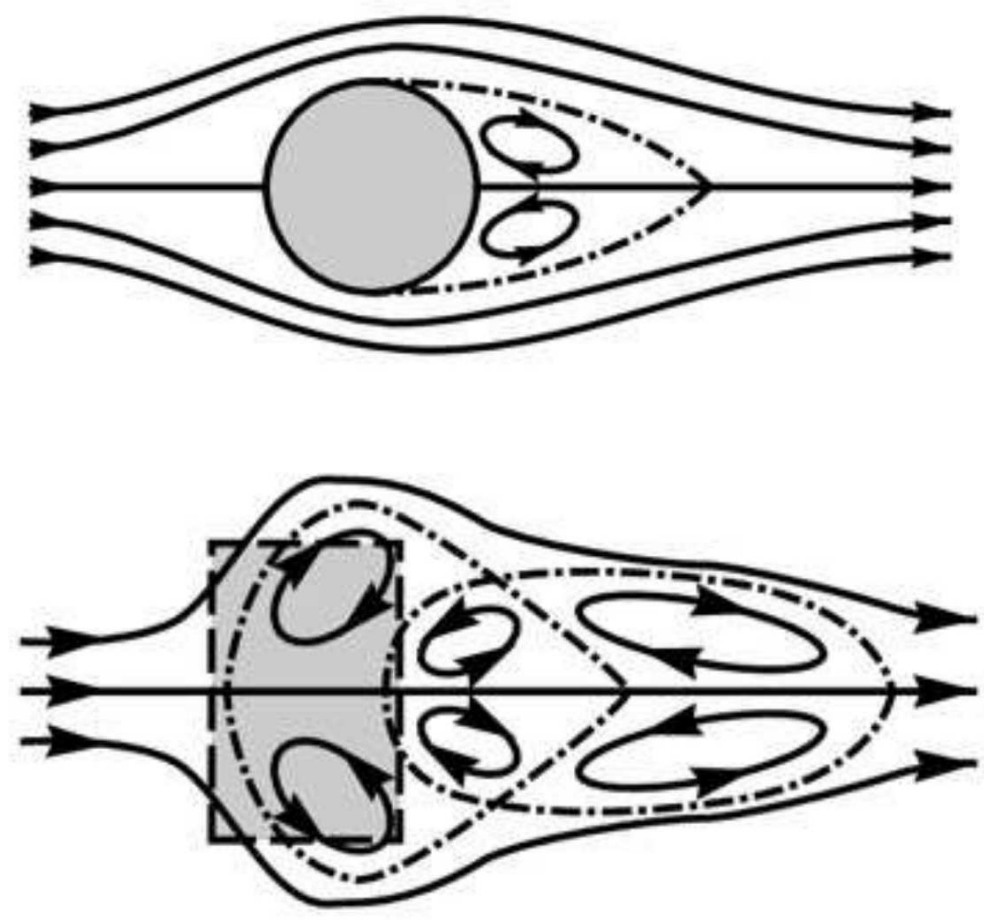

Figure 1: Similarity concepts of flow around solid (Pier, 2002) (gray area, -top) and magnetic obstacle (gray area, -bottom). The attached vortices are generated in the wake of the solid obstacle, while in the wake of the magnetic obstacle, due to localised effects of the Lorentz force, inner (first pair), connecting (second pair) and attached (third pair) can be formed, Votyakov et al. (2008). 


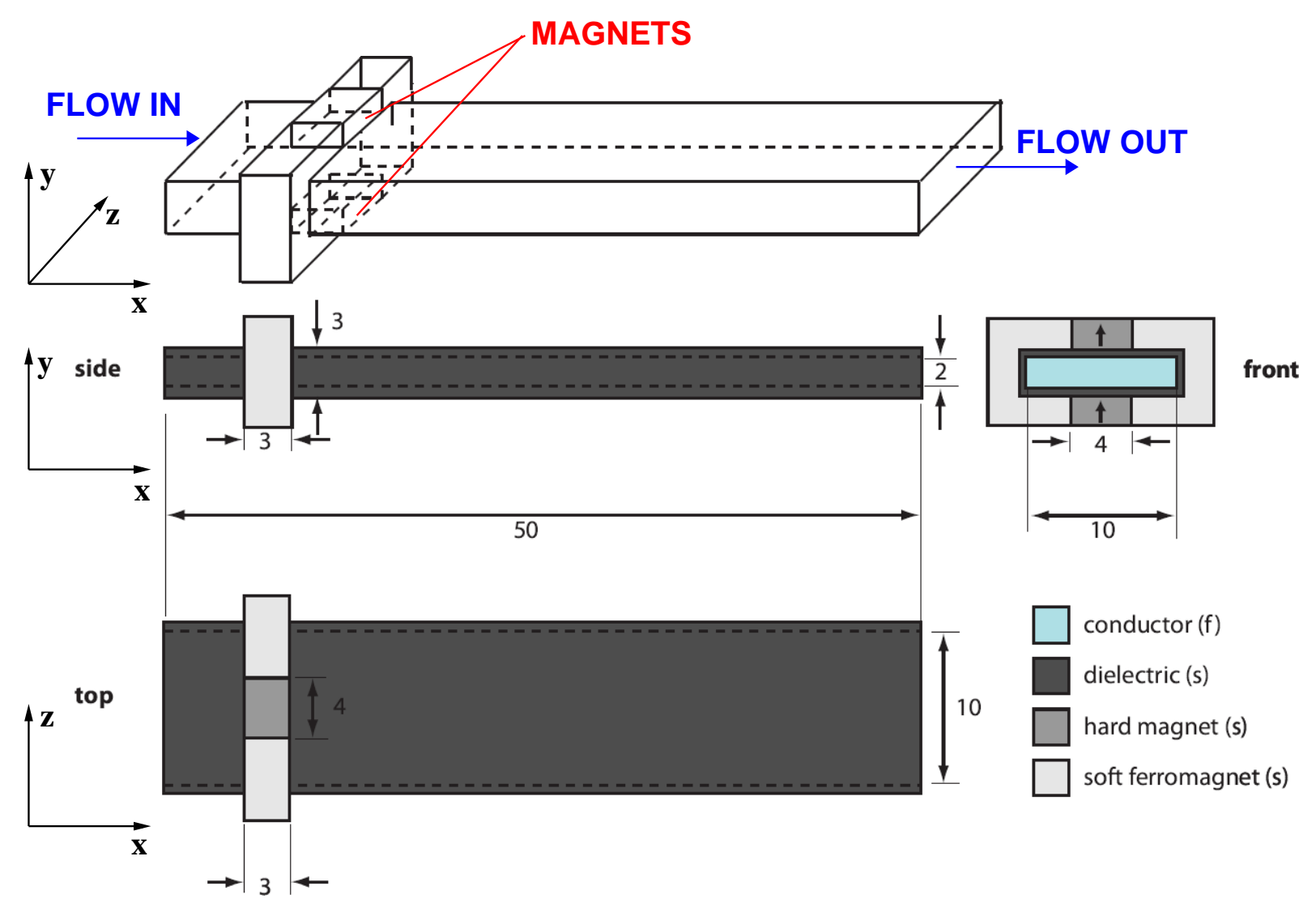

Figure 2: A sketch of the rectangular duct configuration with a locally imposed magnetic field (permanent magnets) and electrically insulated walls with adopted coordinate system: side ( $x-y$ plane), top $(x-z$ plane $)$ and front ( $z-y$ plane) view. Note that all length scales are non-dimensionalised with a half duct height. The direction of magnetisation of the permanent magnets is indicated by arrows in the front view. The flow direction is from left to right - in the $x$ - direction. 

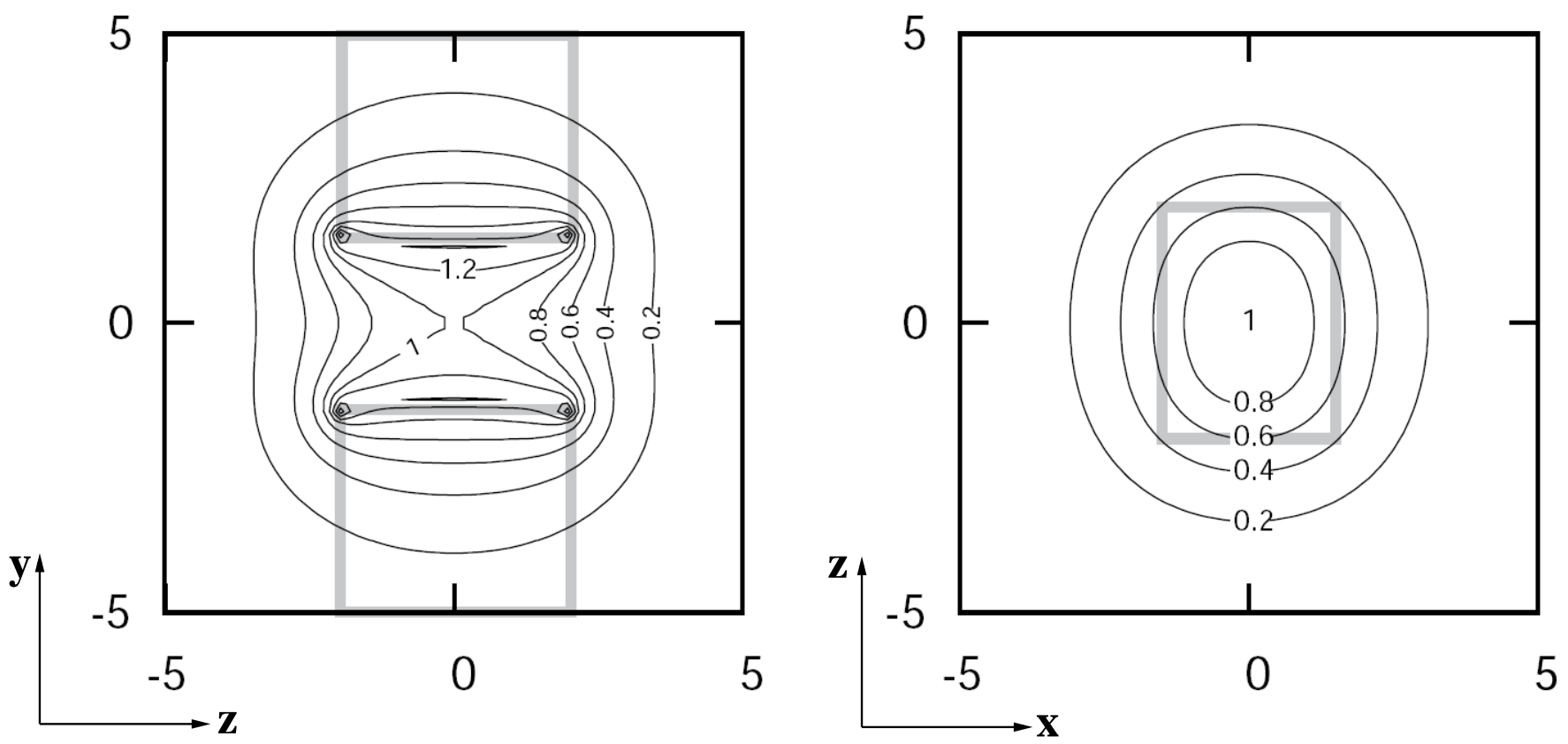

Figure 3: Contour plot of the magnetic field $(|B|$ in $\mathrm{T})$ normalised to the central value between the magnetic pair: front view ( $z-y$ plane) -left, top view of the central plane $(x-z$ plane $)$ - right. All length scales are non-dimensionalised with a half duct height. Gray outlines indicate the magnet edges. 
Case A: $R e=100, N=4, H a=20$

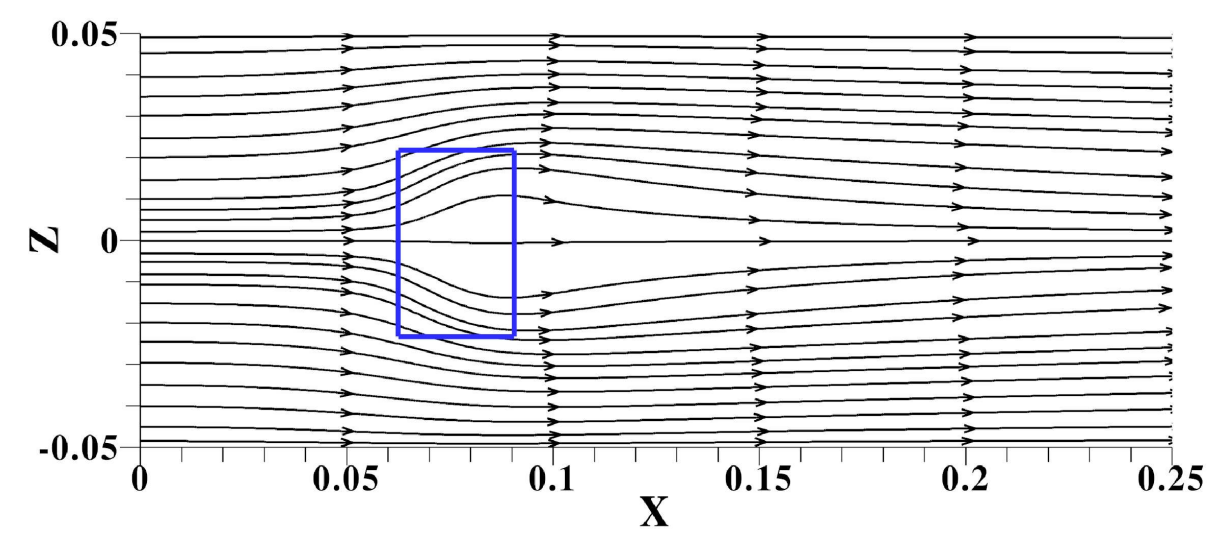

Case B: $R e=100, N=11.25, H a=33.5$

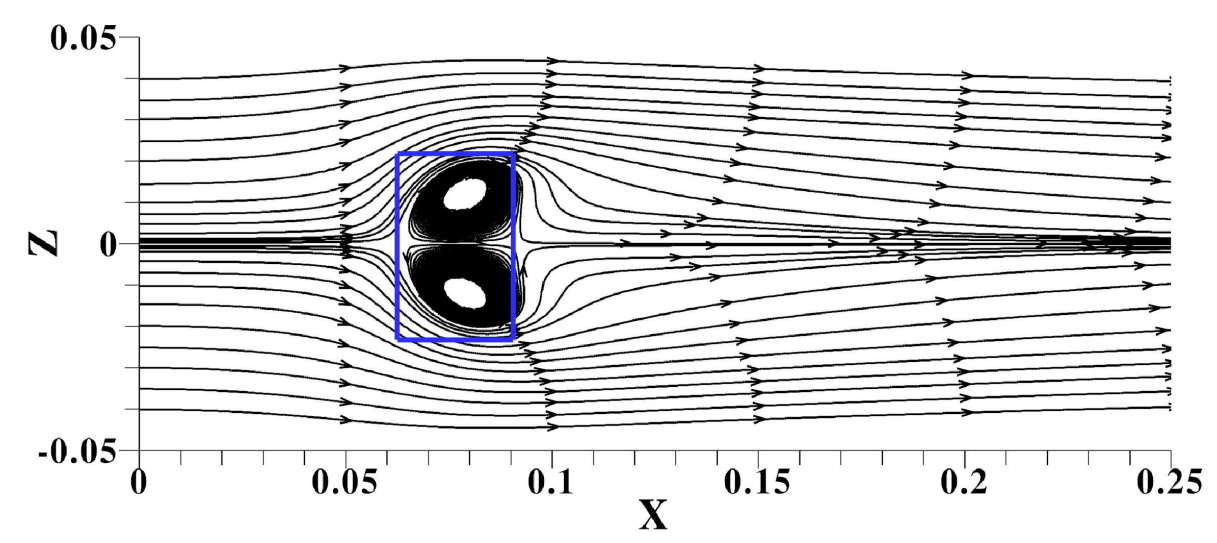

Case C: $R e=400, N=11.25, H a=67$

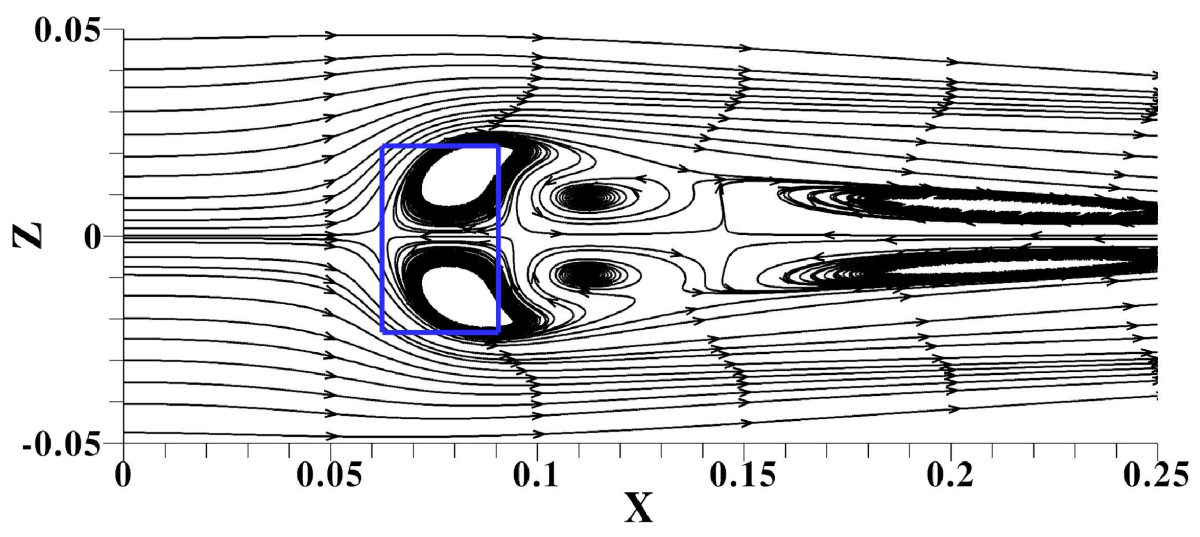

Figure 4: Vortical patterns in a stable flow regime for a low Reynolds number $(R e=100,400)$ around a magnetic obstacle with different strengths of the imposed magnetic field $(N=4,11.25)$ inside a rectangular duct with electrically-insulating walls, visualised by stream-traces in the central horizontal plane. The location of the permanent magnets is indicated by a blue rectangle. 


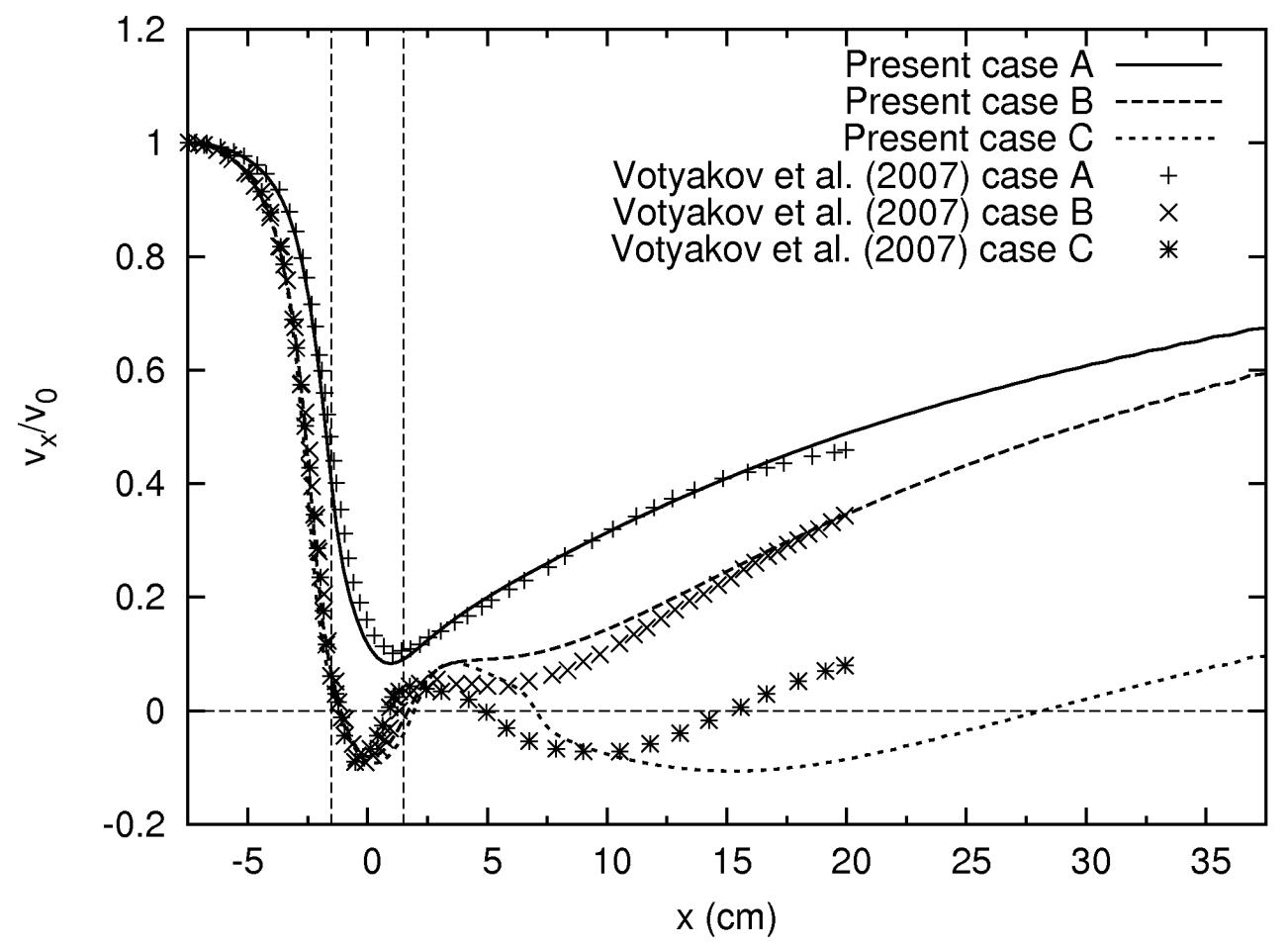

Figure 5: The non-dimensionalised profiles of the streamwise velocity along the centreline in the duct middle plane for three characteristic steady flow patterns: Case A - $R e=100, N=4$; Case $\mathrm{B}-R e=100$, $N=11.25$; Case C $-R e=400, N=11.25$; Comparisons with results obtained in Votyakov et al. (2007). Dashed vertical lines indicate borders of the magnetic obstacle. Note that the origin of the coordinate system is now moved to the magnet centre. $V_{0}$ is maximum of the inlet velocity. 

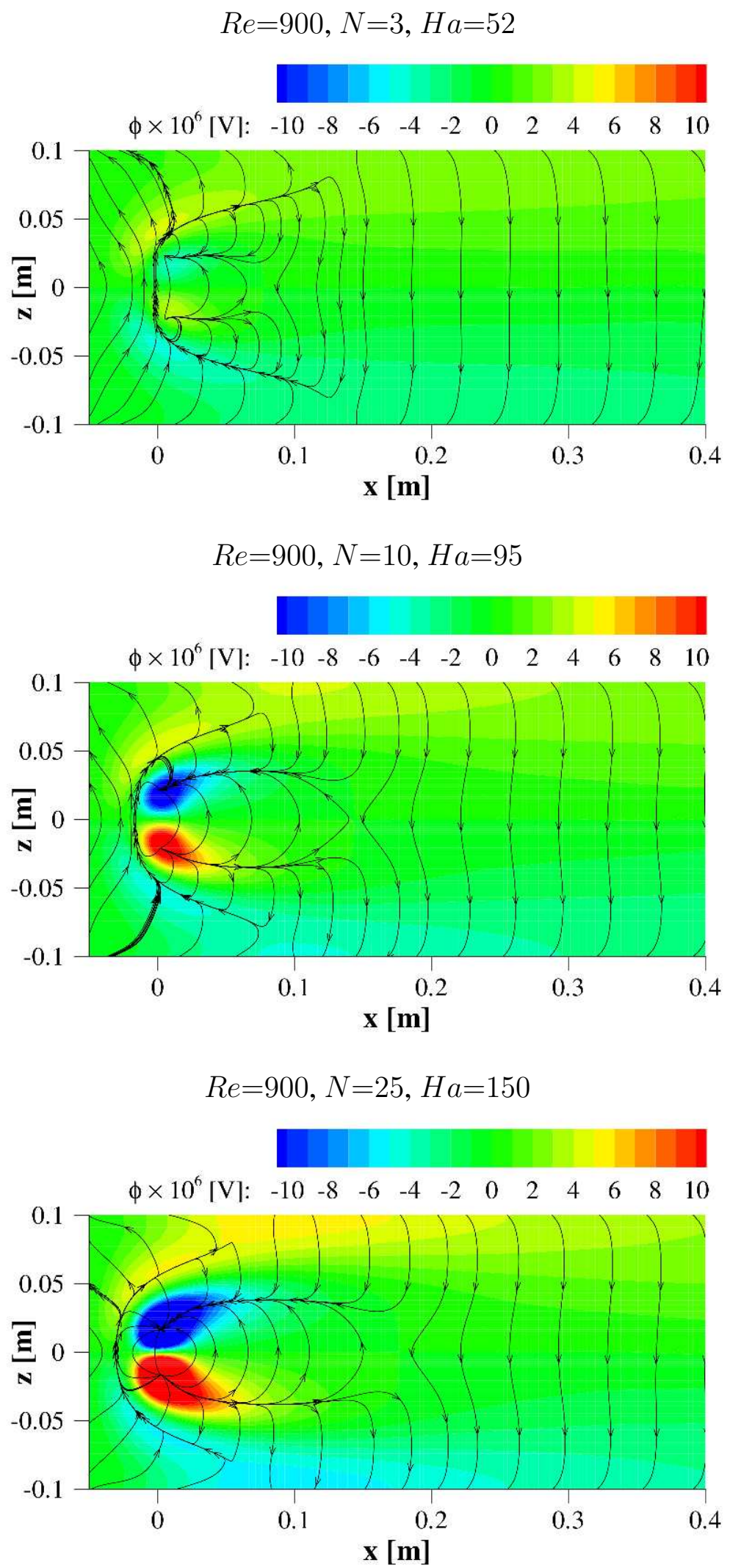

Figure 6: Instantaneous snapshots (for an arbitrary time instant) of contours of the electric potential $(\phi)$ and the total-electric current lines $(\mathbf{J})$ in the central horizontal plane for different strengths of the imposed magnetic field, $N=3,10$ and 25 , respectively. 


$$
R e=900, N=3, H a=52
$$
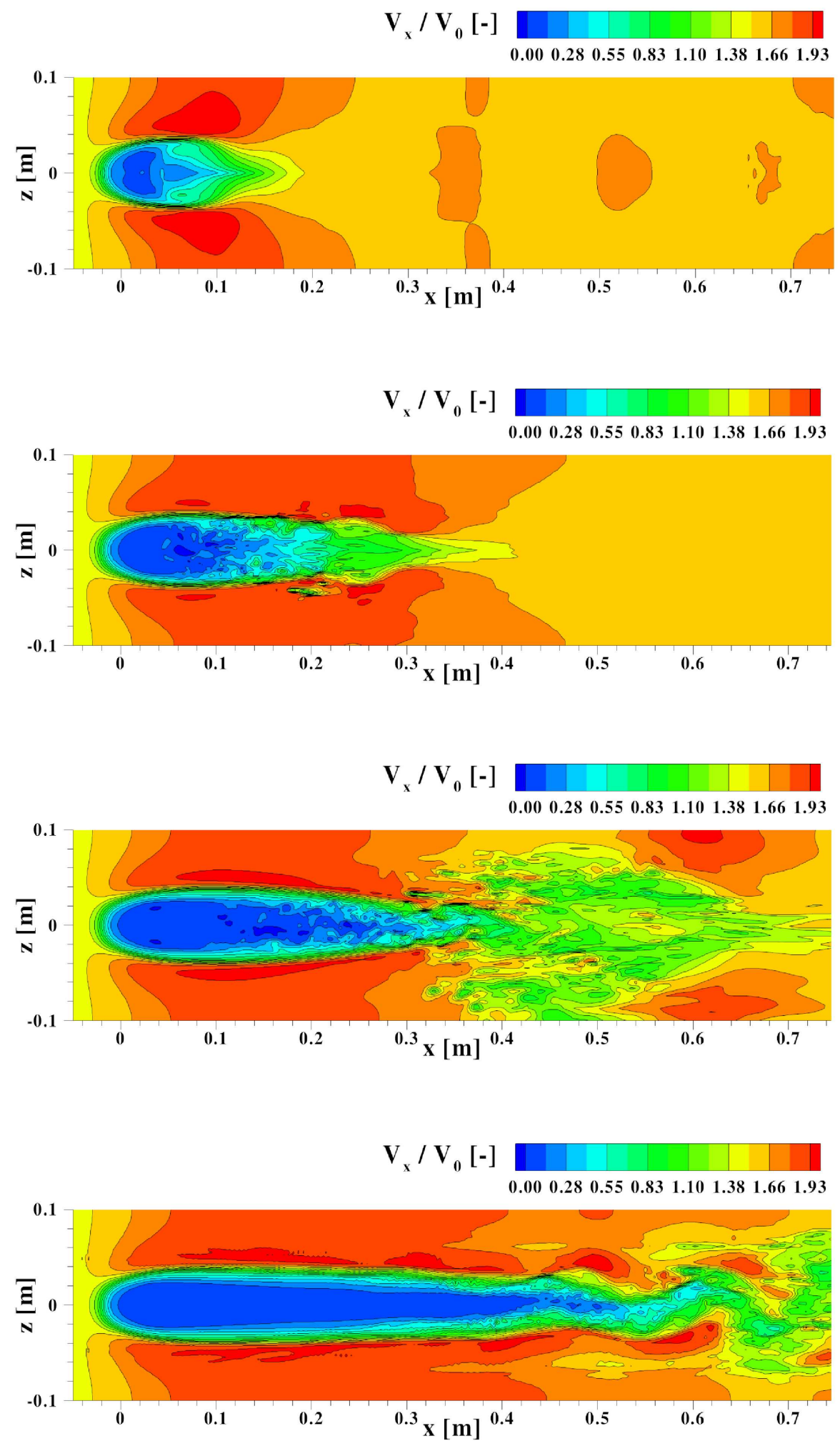

Figure 7: Time evolution of the flow instabilities for $R e=900$ and $N=3$. Contours of the nondimensional streamwise velocity $\left(V_{x} / V_{0}\right)$ in the central plane $(y=0 \mathrm{~m})$ at different time instants: $t=5$, $25,100,1000 \mathrm{sec}$. The characteristic flow-through time is $t^{*}=20 \mathrm{sec}$. 


$$
R e=900, N=10, H a=95
$$
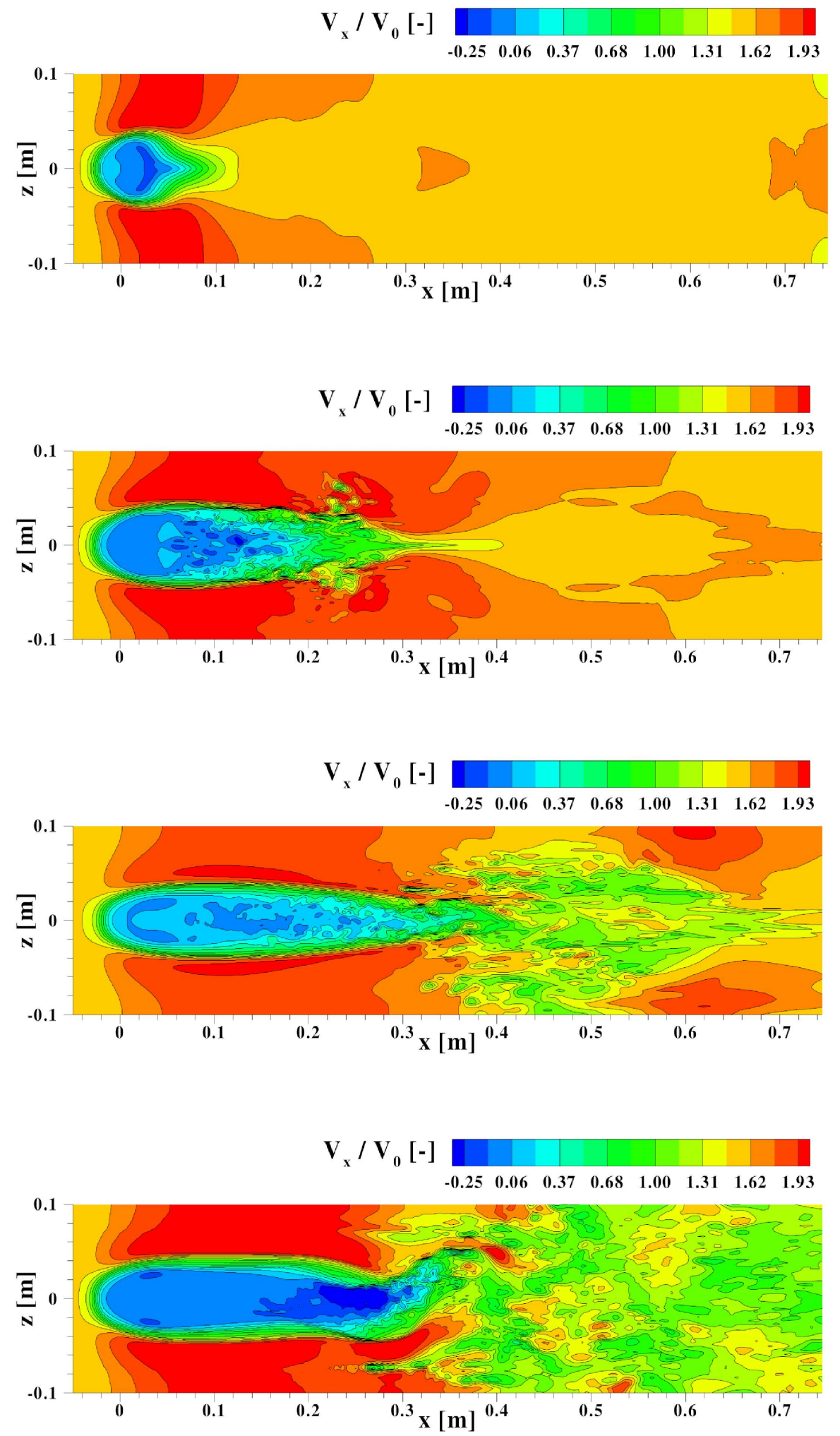

Figure 8: As in previous figure, only for $R e=900, N=10$. 

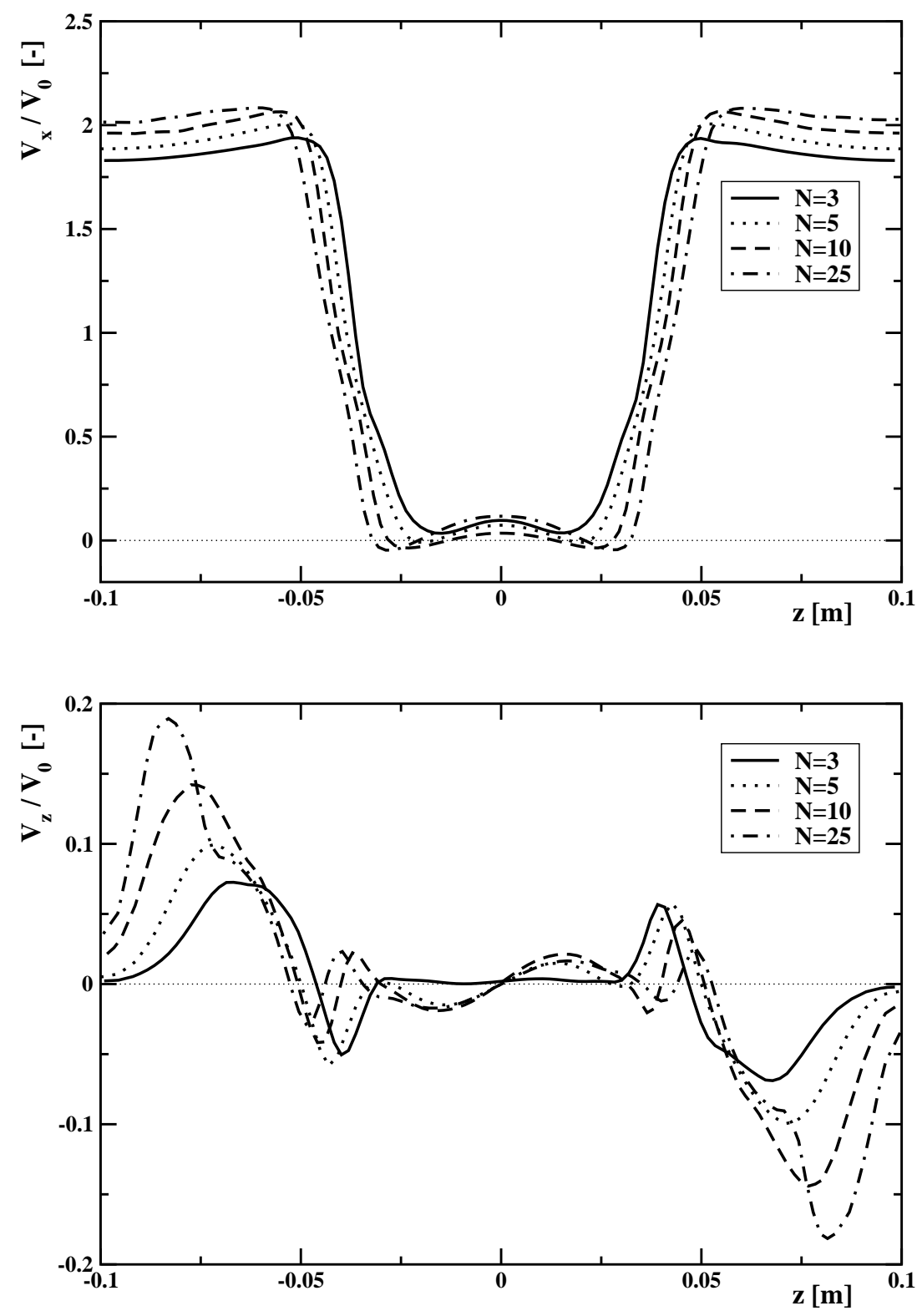

Figure 9: The non-dimensionalised profiles of the streamwise $\left(V_{x} / V_{0}\right)$ and spanwise $\left(V_{z} / V_{0}\right)$ velocity in the central horizontal plane $(y=0 \mathrm{~m})$ at $x=0.07 \mathrm{~m}$ - for an arbitrary time instant. 

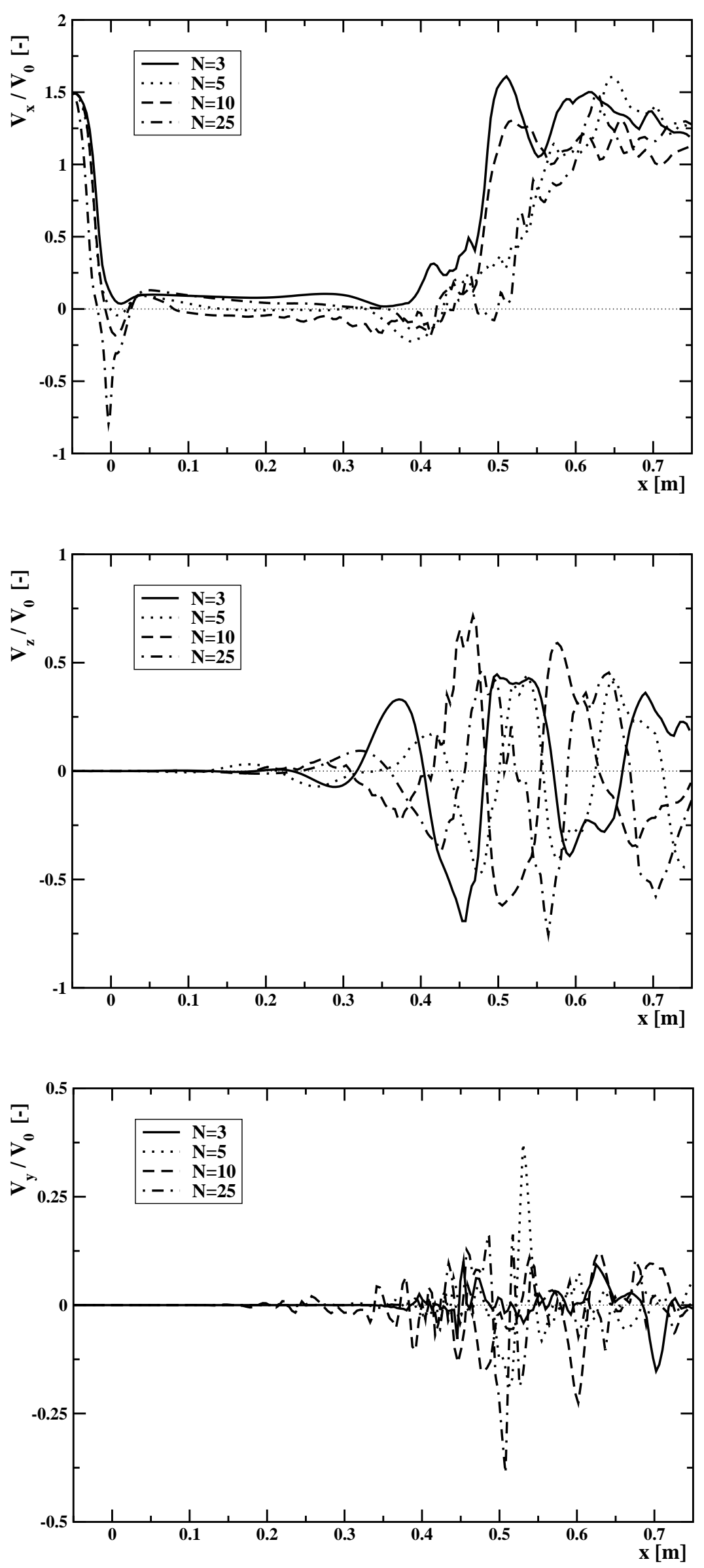

Figure 10: The non-dimensionalised profiles of the velocity components along the channel centreline $y=z=0 \mathrm{~m}) . R e=900$ and different values of $N=3,2610,25$ - for an arbitrary time instant. 
$\operatorname{MON} 1(x=0.15 \mathrm{~m}, y=0 \mathrm{~m}, z=0 \mathrm{~m})$
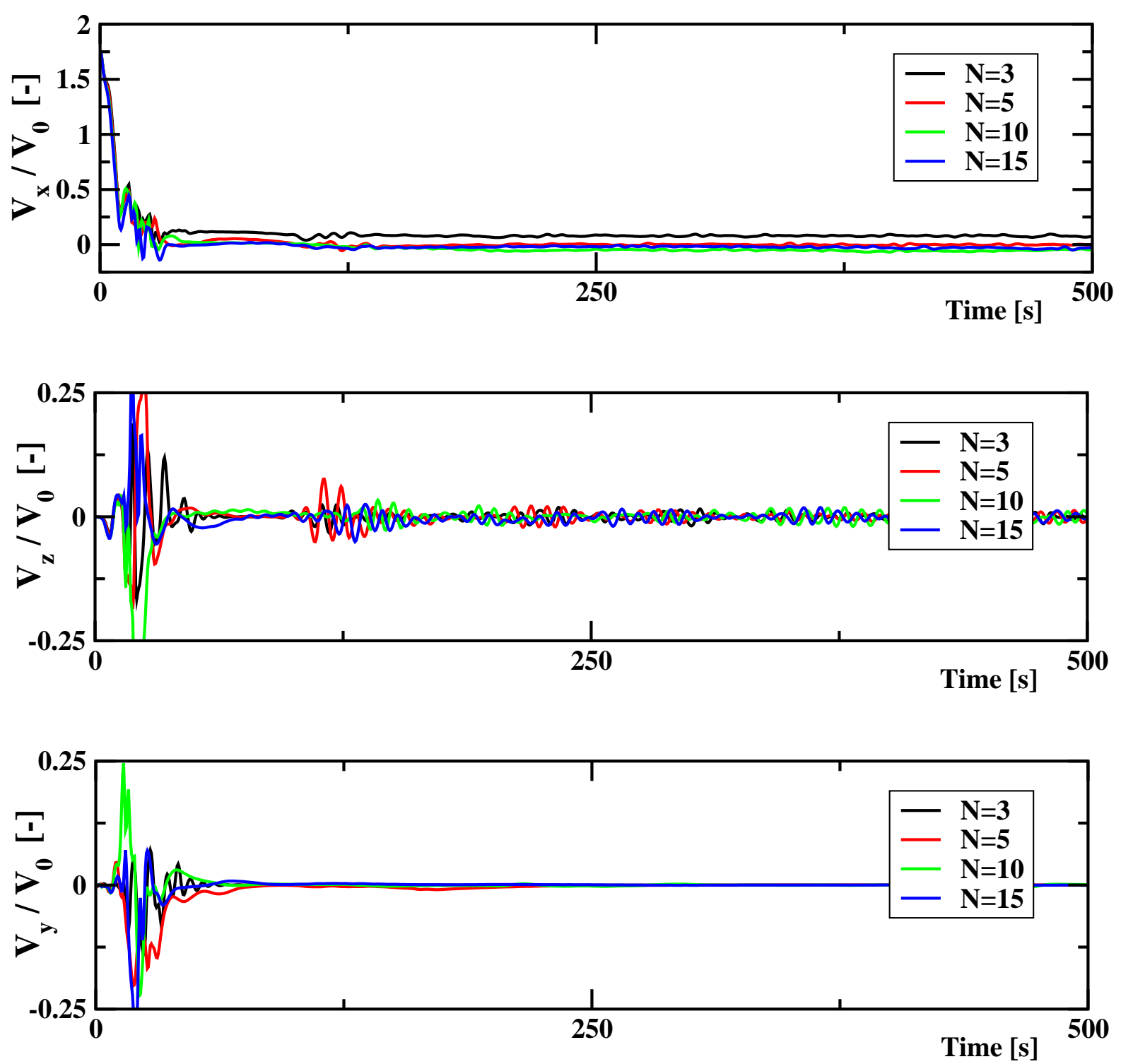

Figure 11: The time-series of the velocity components at monitoring point $(0.15,0 ., 0.) \mathrm{m}$, for different values of the interaction parameter $(N=3, \ldots, 15)$ and fixed value of the Reynolds number $R e=900$. 


$$
\operatorname{MON} 2(x=0.35 \mathrm{~m}, y=0 \mathrm{~m}, z=0 \mathrm{~m})
$$
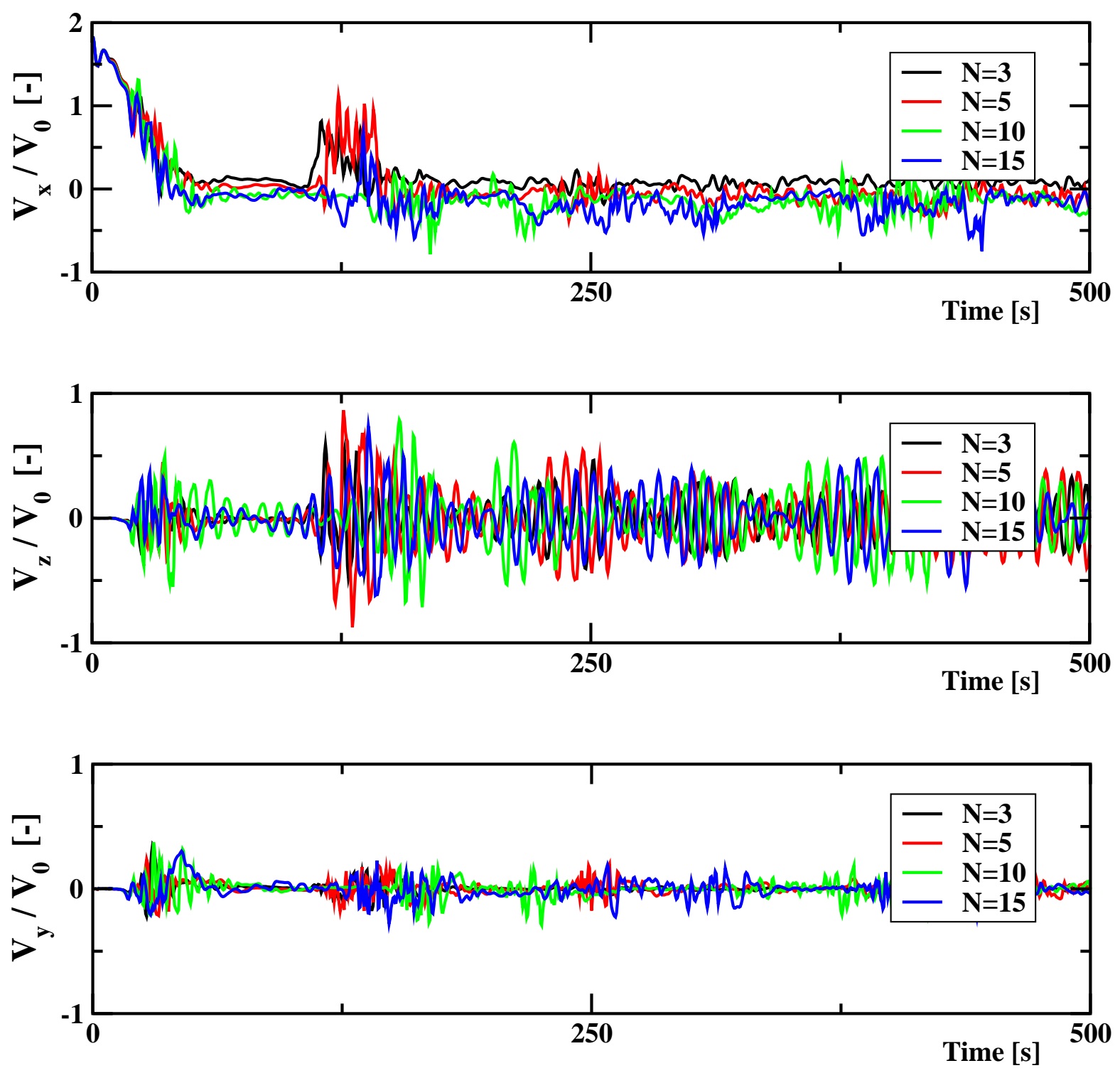

Figure 12: Same as in the previous figure only at monitoring location $(0.35,0 ., 0.) \mathrm{m}$. 

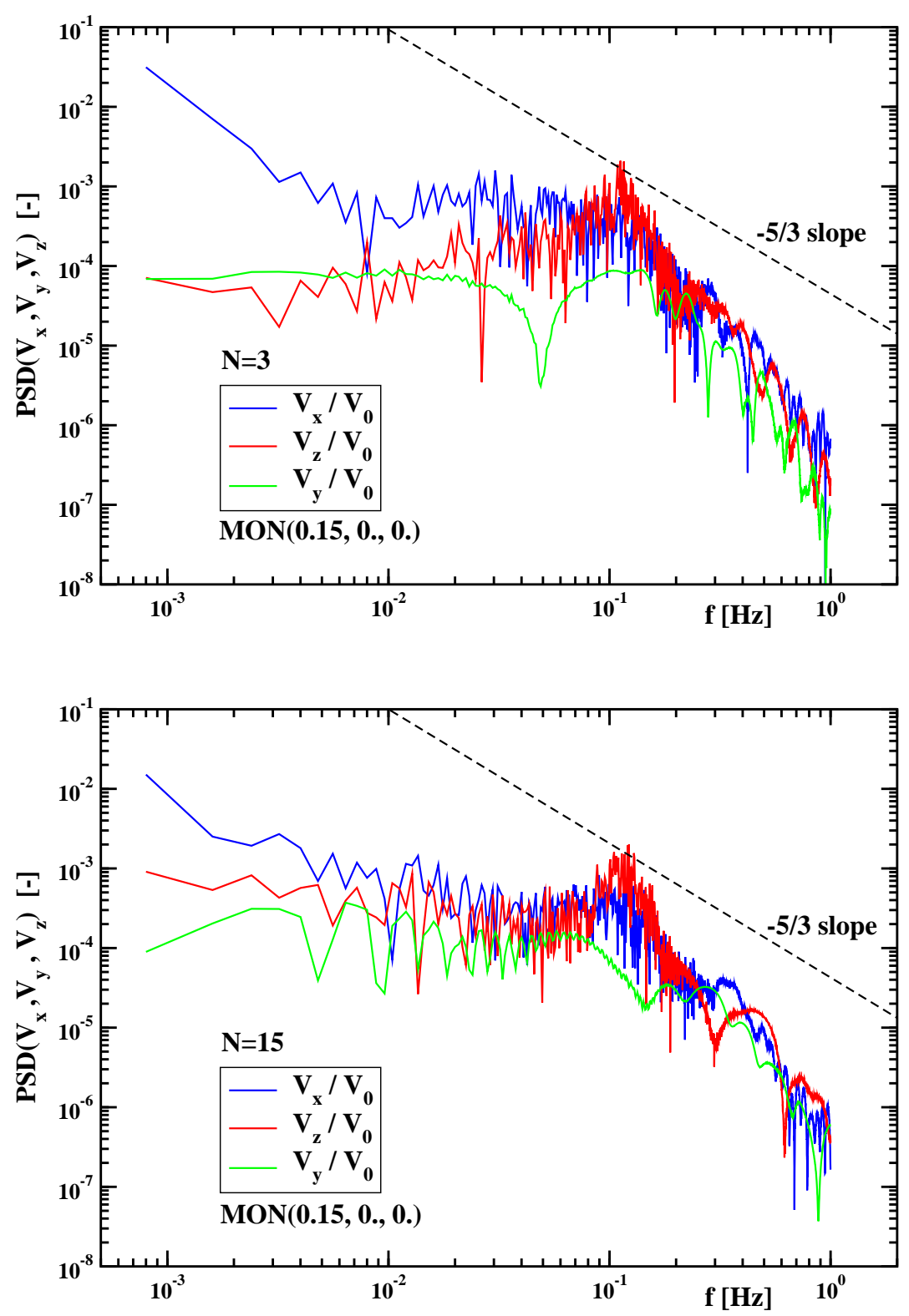

Figure 13: The power spectra of the non-dimensionalised velocity components at a characteristic location in the central horizontal plane $(0.15,0 ., 0.) \mathrm{m}$ for two values of the interaction number, $N=3$ and 15 . The time step used was $\Delta t=0.5 \mathrm{sec}$. 

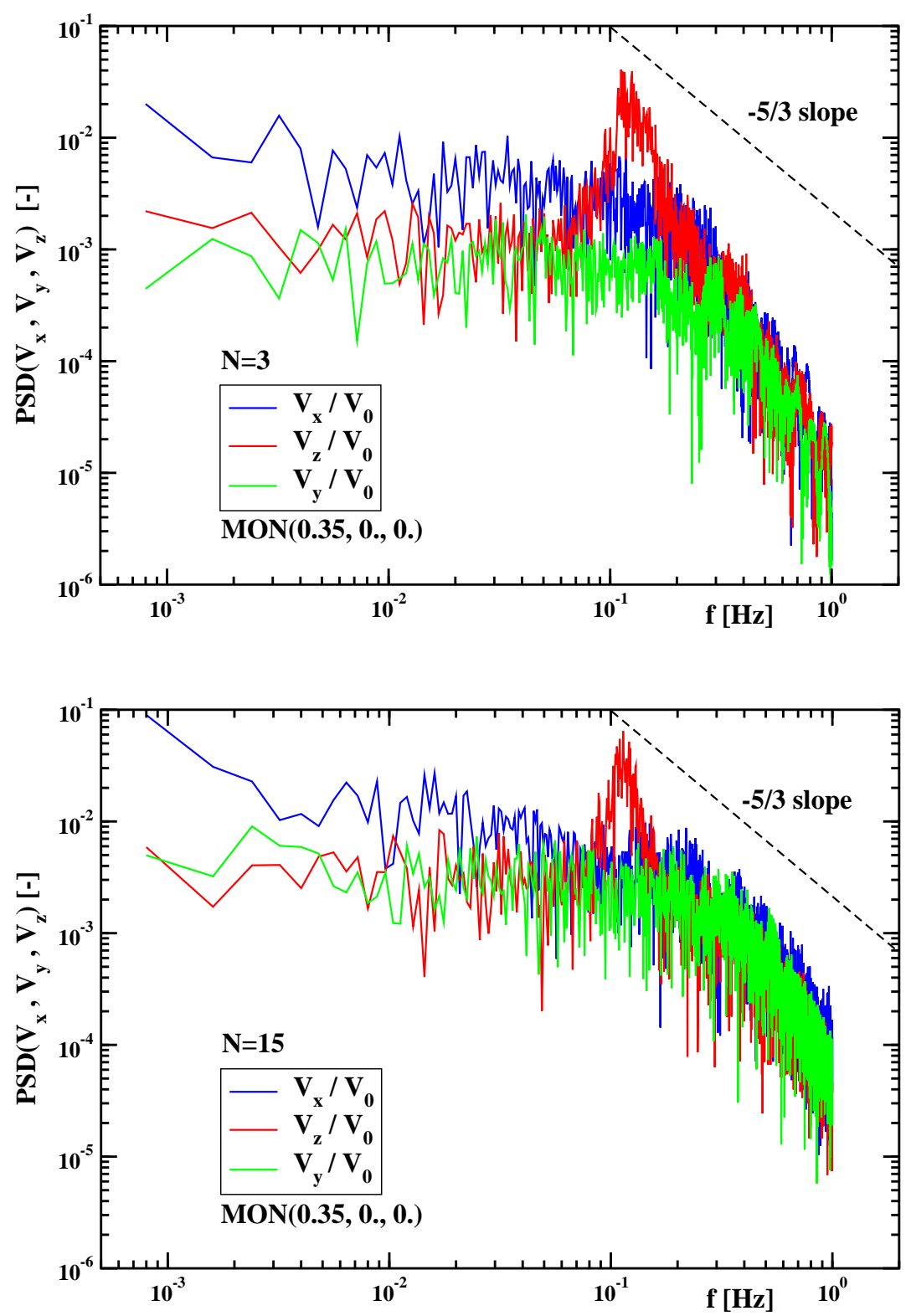

Figure 14: Same as in the previous figure, only at $(0.35,0 ., 0.) \mathrm{m}$. 

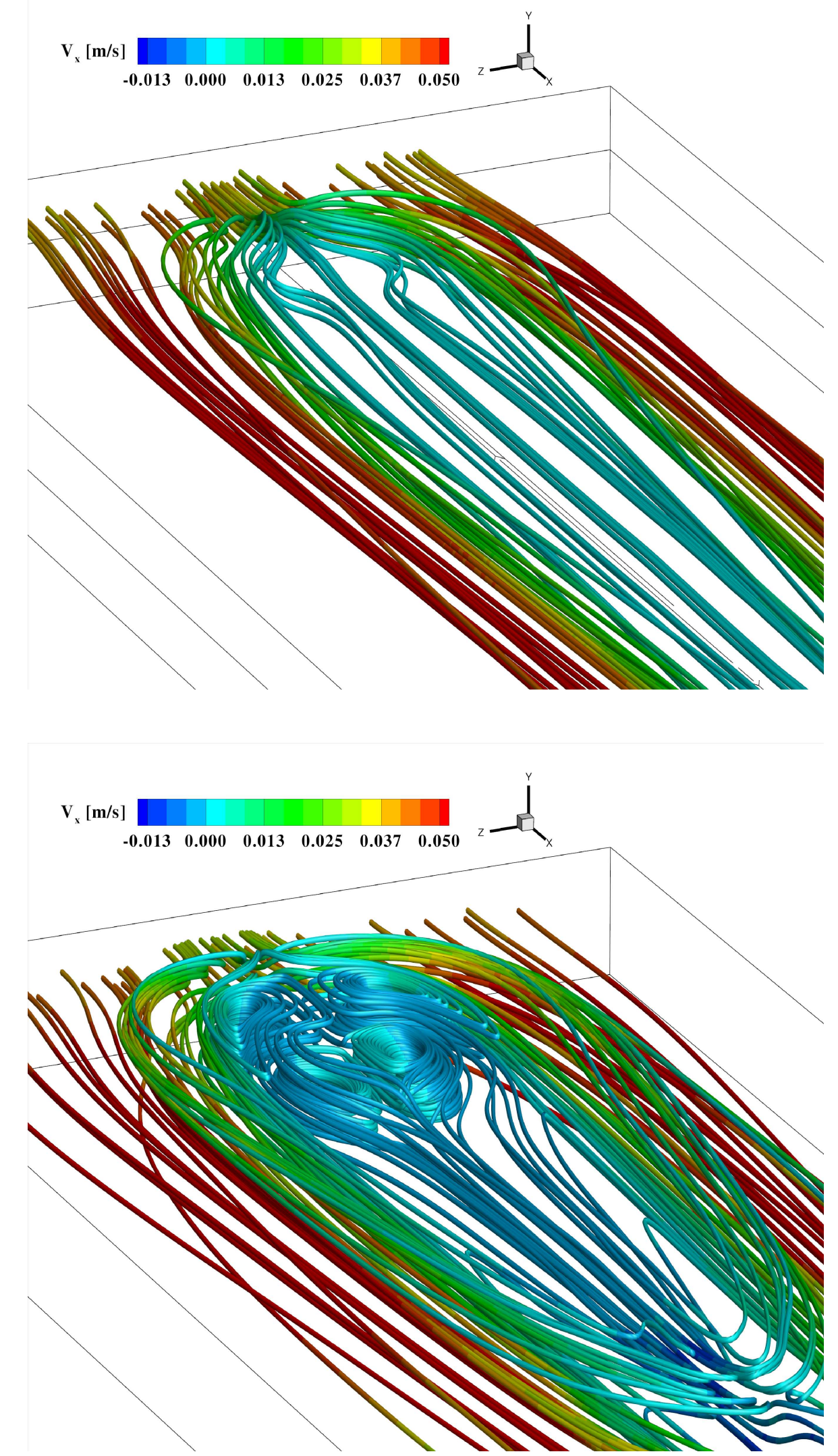

Figure 15: A zoom-in of 3D streamtraces (coloured by streamwise velocity) of long-term averaged velocity fields for $R e=900$ and $N=3$ (-top) and $N=10$ (-bottom). 

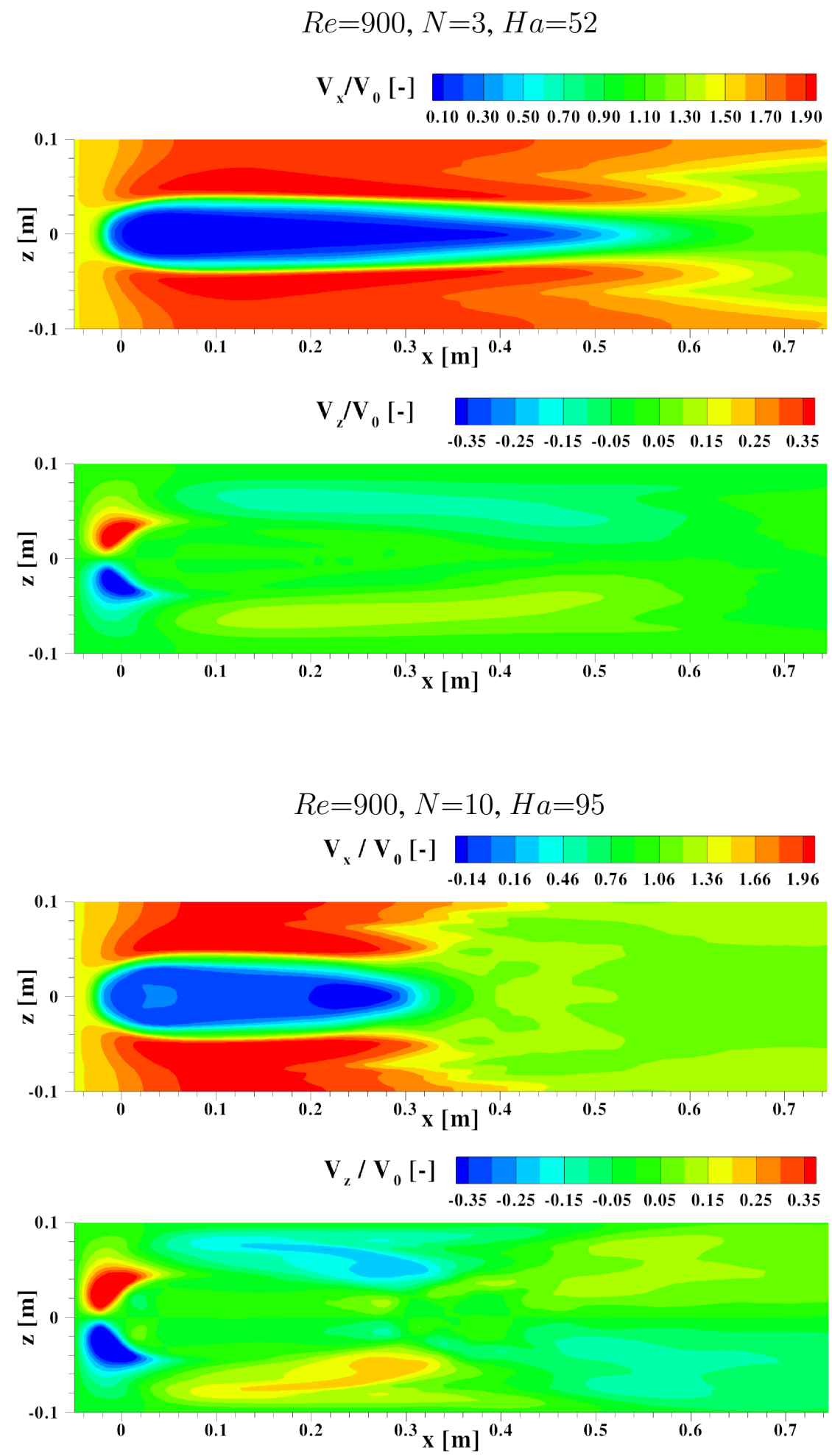

Figure 16: Contours of the long-term averaged non-dimensional streamwise and spanwise velocity components in the central horizontal plane $(y=0 \mathrm{~m})$ for fixed value of $R e=900$ and two different values of $N=3$ (-top) and $N=10$ (-bottom). 


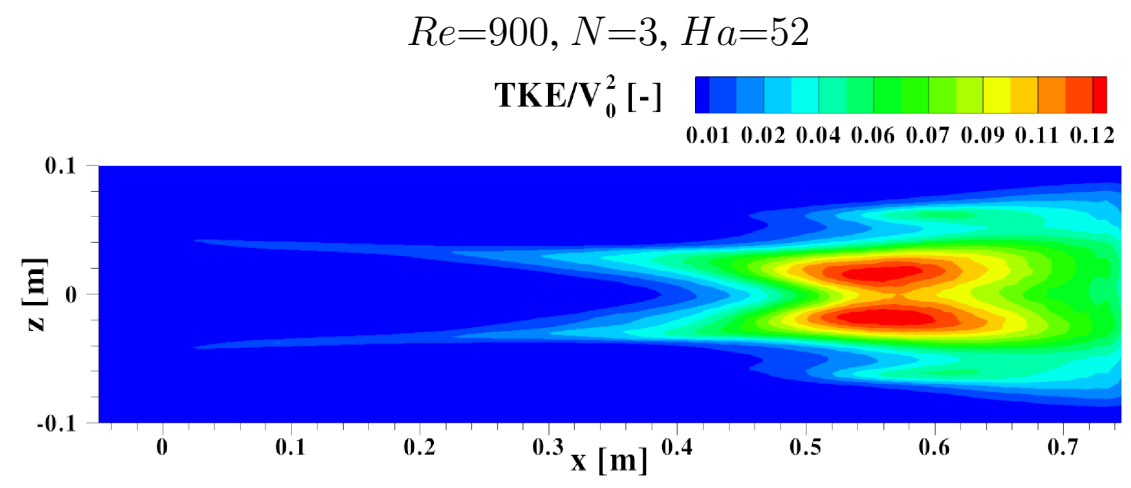

$$
R e=900, N=10, H a=95
$$

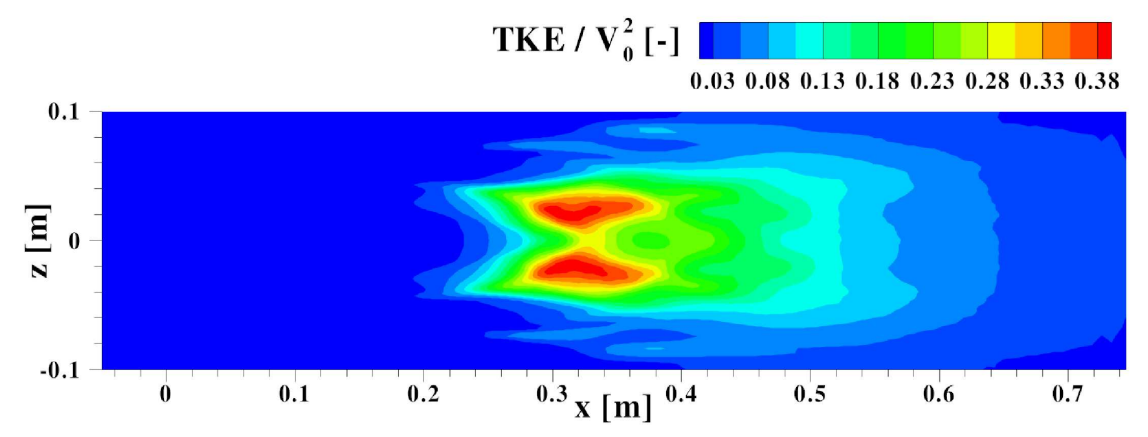

Figure 17: Contours of the non-dimensional turbulent kinetic energy (TKE) in the central horizontal plane $(y=0 \mathrm{~m})$ for the fixed value of $R e=900$ and two different values of the magnetic interaction parameter $N=3$ (-top) and $N=10$ (-bottom). 


$$
R e=900, N=3, H a=52
$$
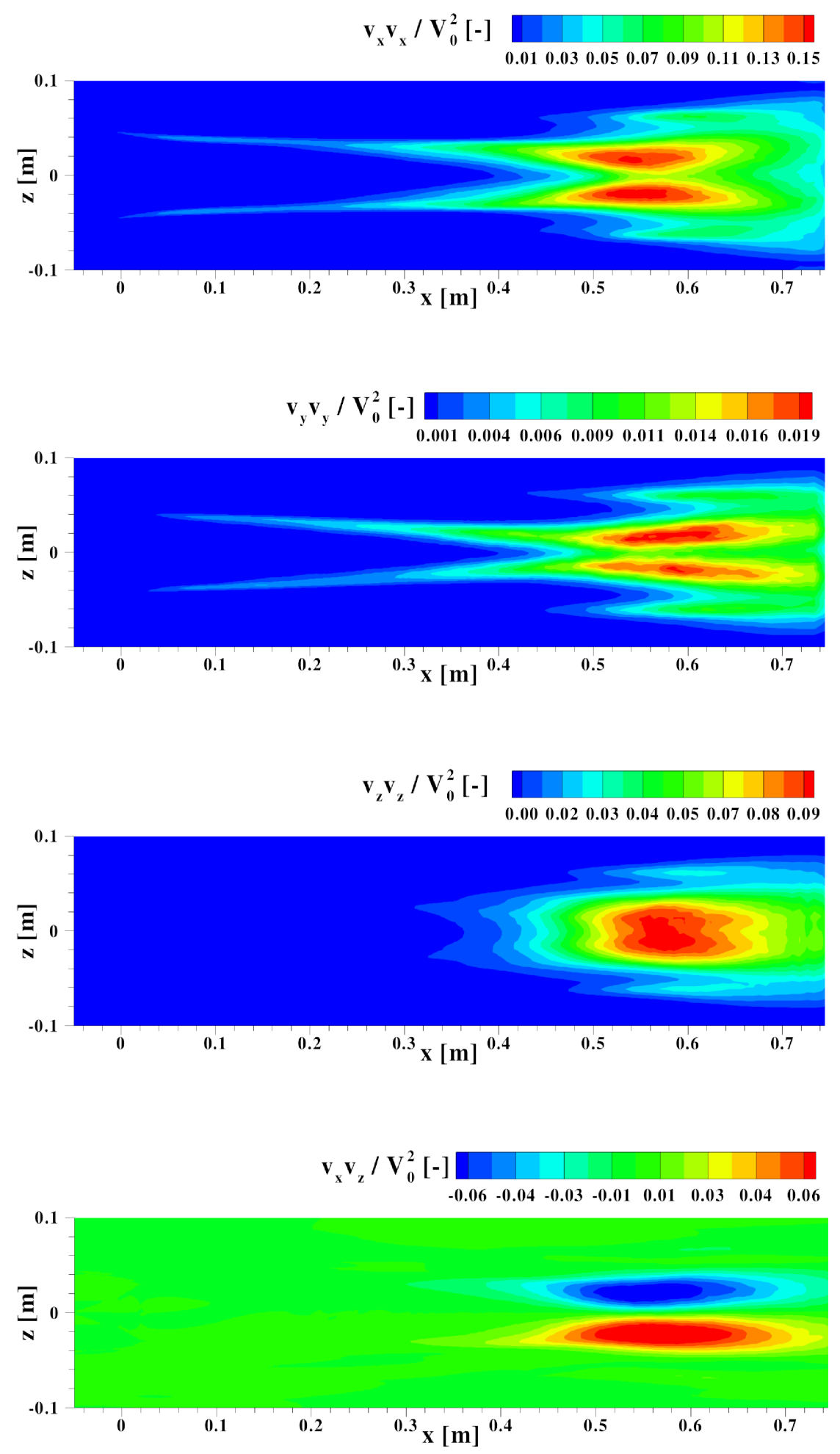

Figure 18: Contours of the normal and shear turbulent stresses in the central horizontal plane $(y=0 \mathrm{~m})$, $R e=900, N=3$. 


$$
R e=900, N=10, H a=95
$$
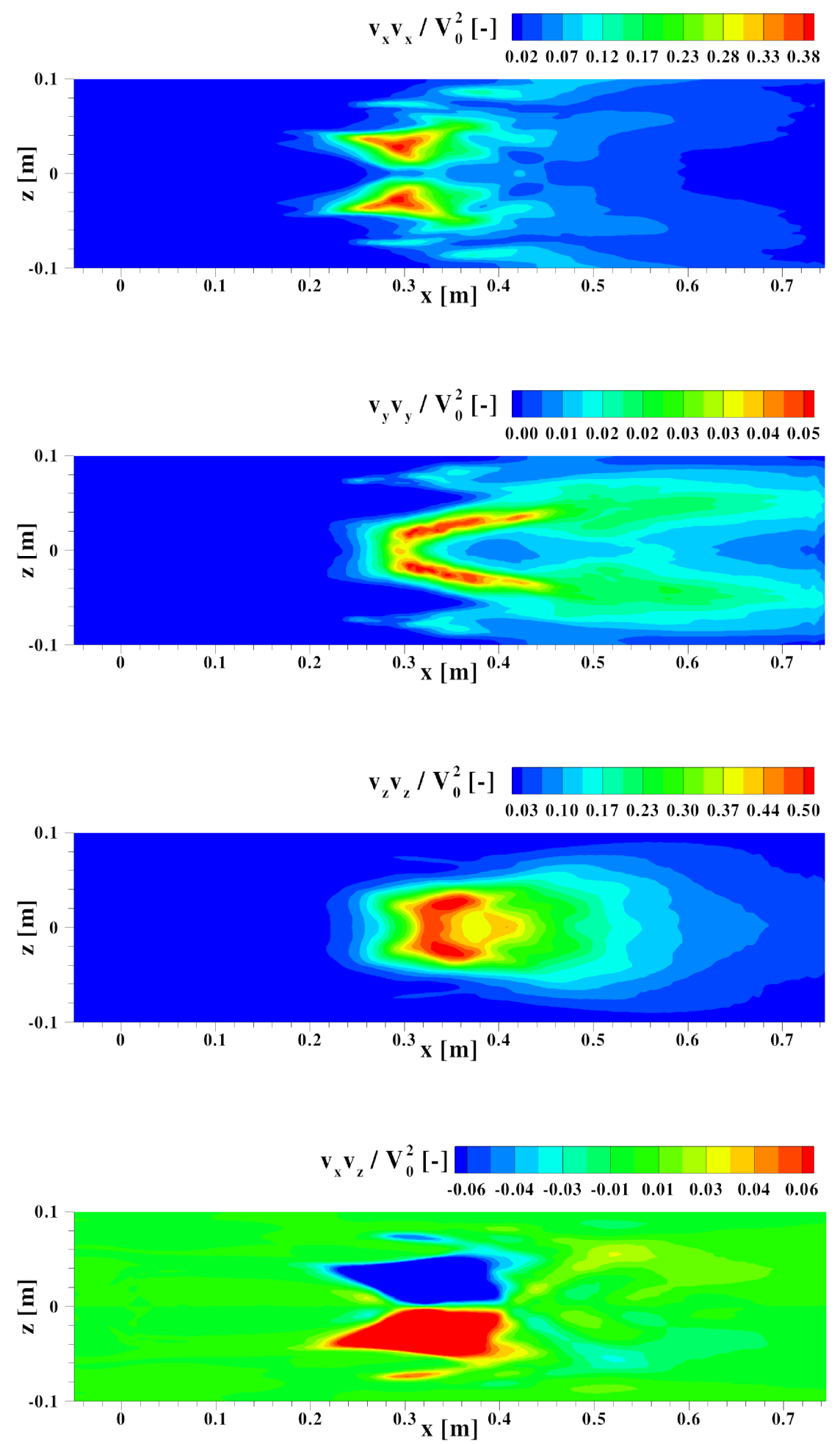

Figure 19: Same as in the previous figure, only for $N=10$. 